\title{
Study of the 1D lattice Boltzmann shallow water equation and its coupling to build a canal network
}

\author{
Pham van Thang ${ }^{(1)}$, Bastien Chopard ${ }^{(2)}$, Laurent Lefèvre ${ }^{(3)}$, \\ Diemer Anda Ondo ${ }^{(4)}$, Eduardo Mendes $^{(4)}$ \\ (1) GIPSA-Lab, Université de Grenoble, France \\ (2) University of Geneva, Switzerland \\ (3) LAGEP, CNRS - Université Claude Bernard Lyon 1, France \\ (4) LCIS-ESISAR, Grenoble INP, France
}

June 9, 2010

\begin{abstract}
The D1Q3 lattice Boltzmann (LB) shallow water equation is analyzed in detail and compared with other numerical schemes. Analytical results are derived and used to discuss the accuracy and stability of the model. We show how such D1Q3 LB models for canal reaches may be easily coupled with various hydraulic interconnection structures to build models of complex irrigation networks.
\end{abstract}

\section{Introduction}

Generally, an irrigation network consists of a primary open-air canal connected to secondary canals and/or pressurized network of water distribution. Canals consist of several long reaches (usually several kilometers long) separated by engineering works (like sliding gates for instance) $[8,4]$. The open-channel hydraulic part is the most complex one. Its dynamical behavior is characterized by important time delays (due to water transport), wave superposition effects and strong nonlinearities (mainly around the works). The overall network has to be carefully managed in order to supply the various water flow demands without violating strict water level constraints at several places along the reaches and near the hydraulic works. This complex control and optimization problem requires efficient and reliable numerical algorithms to describe the open-air hydrodynamics.

Within the long reaches with uniform sections, 1D shallow water model are usually used successfully, whereas 2D/3D models are often required to describe nonlinear turbulent flows as well as important erosion/sedimentation effects near the gates. Recently we have proposed a bi-fluid lattice Boltzmann (LB) model that describes the flow near a gate, with or without sediment transport and 
erosion [14], fully resolving all the components of the velocity flow. However, this detailed model is computationally demanding and should only be used in the regions where the vertical component of the flow velocity plays an essential role.

In this paper, we use a lattice Boltzmann (LB) approach to solve the 1D shallow water (SW) equation and its coupling with other models. Whereas 2D LB-SW models have been considered in several papers [16, 5, 22], the 1D model is -to our knowledge- only investigated in one article by Frandsen [6].

In what follows we present a detailed analytical discussion of the accuracy and stability of the 1D model, as well as a comparison with other time-dependent numerical solvers. We show that the LB-SW model is accurate, fast and rather stable. We also consider the coupling between several 1D models separated by gates whose behaviors are described by a phenomenological equation. More advanced coupling will be discussed briefly and described in a forthcoming publication.

\section{The shallow water equation}

\subsection{Governing equations}

We consider here the water flow in a rectangular open channel with slope, $I$, and width, $B$, as represented in (Fig.1). The flow and level dynamics of water in such a channel is usually modelled by the shallow water equations which are derived from the conservation laws of mass and momentum, using some assumptions on the flow. These assumptions are that the slope is small, the length of the reach is assumed sufficiently large compared to the water level height, the pressure is assumed to be hydrostatic and the fluid is incompressible. Finally, internal viscosity effects are neglected. The Saint-Venant (or shallow water) equations are then

$$
\begin{aligned}
\partial_{t} h+\partial_{x}(h u) & =0 \\
\partial_{t}(h u)+\partial_{x}\left(\frac{1}{2} g h^{2}+h u^{2}\right) & =F
\end{aligned}
$$

where $h$ denotes the water depth, $u$ the depth-averaged horizontal velocity of the flow, and $g$ the gravitational acceleration. The force term, $F=g h(I-J)$, accounts for the bed slope, $I$, and the bed friction, $J$, where $I=\partial h_{b} / \partial x$ with $h_{b}$ the bed height and $J$ is modelled with the classical Manning formula [8]:

$$
J=\frac{n^{2} u^{2}}{\left(\frac{B h}{B+2 h}\right)^{4 / 3}}
$$

with $n$ the Manning coefficient and $B$ the width of the canal. 


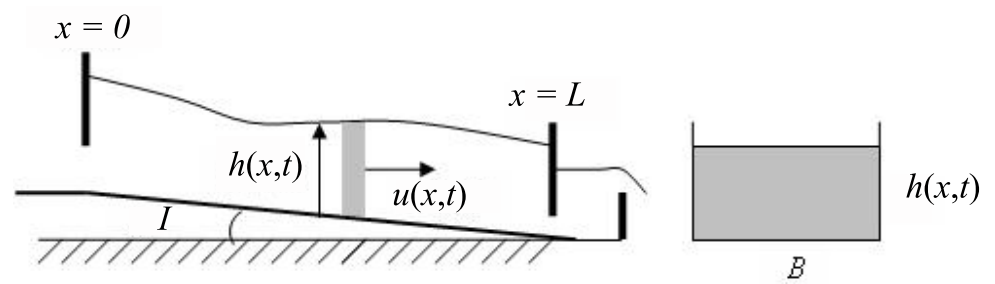

Figure 1: Longitudinal (left) and lateral (right) views of an open rectangular hydraulic channel

\subsection{Lattice Boltzmann model}

The lattice Boltzmann (LB) method has proven to be a powerful numerical tool to simulate the fluid flows and other physical phenomena [15, 1, 2, 20].

In this method, one considers the dynamics of idealized fluid particles on a lattice. The key quantities in the LB model are the density distributions, $f_{i}(x, t)$, denoting the density of particles entering site $x$ at discrete time $t$ with velocity $v_{i}$. The $v_{i}$ are chosen to match the spatial lattice so that, in one time step, $\Delta t$, particle with velocity, $v_{i}$, arrives at the lattice point at $x+v_{i} \Delta t$. Usually a LB model uses only a small number of velocities, $v_{i}$.

One assumes that the particles entering the same site at the same time with density, $f_{i}^{i n}(x, t)$, collide. As a consequence, a new distribution, $f_{i}^{\text {out }}(x, t)$, of particles results. Then, during the next time step, $t+\Delta t$, the particles emerging from this collision phase move to a new lattice site, determined by their new speeds. Therefore, the dynamics of a LBM consists of the alternation of collision and streaming phases

$$
\begin{array}{ll}
\text { Collision : } & f_{i}^{\text {out }}(x, t)=f_{i}^{\text {in }}(x, t)+\Omega_{i}\left(f^{\text {in }}\right) \\
\text { Streaming : } & f_{i}^{\text {in }}\left(x+v_{i} \Delta t, t+\Delta t\right)=f_{i}^{\text {out }}(x, t)
\end{array}
$$

where $f^{i n}$ denotes the vector of all $f_{i}^{i n}, \Delta x$ is the lattice spacing and $\Delta t$ is the time step. $\Omega_{i}$ is the collision operator, which is commonly defined by the Bhatnagar-Gross-Krook(BGK) model [15]

$$
\Omega_{i}\left(f^{i n}\right)=\frac{1}{\tau}\left(f_{i}^{e q}-f_{i}^{i n}\right)
$$

where $\tau$ is a relaxation time constant and the $f_{i}^{e q}$ are the so-called equilibrium distribution functions. $f_{i}^{e q}$ depend on the physical process to be described. We shall specify its form below, for the shallow water model.

Equations (4) and (5) may be combined to obtain the evolution equation

$$
f_{i}\left(x+v_{i} \Delta t, t+\Delta t\right)=f_{i}(x, t)+\frac{1}{\tau}\left(f_{i}^{e q}-f_{i}\right)
$$

where $f$ stands for $f^{i n}$. 
When an external force, $F$, exists, the lattice Boltzmann equations are modified. Several versions have been proposed in the literature [18, 10, 9, 11]. For a constant force, $F$, the following expression can be used:

$$
f_{i}\left(x+v_{i} \Delta t, t+\Delta t\right)=f_{i}(x, t)+\frac{1}{\tau}\left(f_{i}^{e q}-f_{i}\right)+w_{i} \frac{\Delta t}{c_{s}^{2}} v_{i} F
$$

where $w_{i}$ and $c_{s}$ are parameters that are determined by the geometry of the lattice and chosen to obtain isotropy for the model.

In the present paper, we consider a 1D model for the water flow. A D1Q3 model geometry ( 1 dimension and 3 velocities) has been chosen with the notation of figure 2

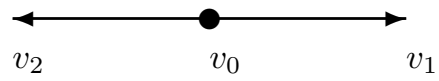

Figure 2: Lattice Boltzmann D1Q3

in which $v_{0}=0, v_{1}=v, v_{2}=-v$ and $v=\Delta x / \Delta t$. In this model, the following values are used for $w_{i}$ and $c_{s}^{2}=\sum_{i>0} w_{i} v^{2}$ :

$$
w_{0}=\frac{2}{3}, \quad w_{1}=w_{2}=\frac{1}{6}, \quad c_{s}^{2}=\frac{v^{2}}{3}
$$

In order to recover the physics of the shallow water equations, the equilibrium distribution functions must satisfy the following three conditions, expressing mass and momentum conservation, as well as the desired form of the momentum tensor

$$
\begin{aligned}
\sum_{i} f_{i}^{e q} & =h \\
\sum_{i} v_{i} f_{i}^{e q} & =h u \\
\sum_{i} v_{i}^{2} f_{i}^{e q} & =\frac{1}{2} g h^{2}+h u^{2} \equiv \Pi^{e q}
\end{aligned}
$$

where $h$, the water level, and $u$, the velocity, are defined as:

$$
h=\sum_{i} f_{i}, \quad h u=\sum_{i} v_{i} f_{i}
$$

When equations (9-11) hold, the equilibrium distribution functions are uniquely determined by the macroscopic variables $h$ and $u$. One gets

$$
\begin{aligned}
f_{0}^{e q} & =h-\frac{1}{2 v^{2}} g h^{2}-\frac{1}{v^{2}} h u^{2} \\
f_{1}^{e q} & =\frac{1}{4 v^{2}} g h^{2}+\frac{1}{2 v} h u+\frac{1}{2 v^{2}} h u^{2} \\
f_{2}^{e q} & =\frac{1}{4 v^{2}} g h^{2}-\frac{1}{2 v} h u+\frac{1}{2 v^{2}} h u^{2}
\end{aligned}
$$


These equilibrium distribution functions will be used in the next section to show that the shallow water equations dynamics may be recovered from the LB model. A dissipative contribution will be obtained that differs from that proposed in [22] but agrees with the results of [5].

Finally, the force term will be evaluated using several methods. First we consider the centered-scheme proposed by Zhou [22]. This scheme was shown be accurate up to the second-order in the space and time discretization steps [22] but our simulations show that this is not always the case. Zhou's force model assumes an LB eq. (7) with

$$
F_{i}=g \bar{h}_{i}\left(I-\frac{n^{2} \bar{u}_{i}}{\left(\frac{B \bar{h}_{i}}{B+2 \bar{h}_{i}}\right)^{4 / 3}}\right)
$$

where $\bar{h}_{i}=\frac{h(x, t)+h\left(x+v_{i} \Delta t\right)}{2}$ and $\bar{u}_{i}=\frac{u(x, t)+u\left(x+v_{i} \Delta t\right)}{2}$. Therefore a kind of mean force term is thus used, derived from the mean values of the water levels and velocities at the current lattice point and at the "next" lattice point in direction $i$.

Unfortunately, Zhou's expression for the force does not conserve mass locally. As shown below, second order corrections to the mass conservation laws are present, as well as a second order correction to the momentum balance equation. Therefore we will also implement Guo's force model [9], which is the standard way to add an external force in the LB method. It will however change the relation (12) between $h u$ and the $f_{i}^{\prime} s$. The accuracy of Guo's force will be shown to be first order only in an exact calculation.

Finally, we shall also consider a simplified Guo's force model, which turns out to be easier to implement and more accurate than the other two in our benchmarks.

\subsection{Chapman-Enskog expansion}

The hydrodymanic equations associated with the above LB model can be derived from a multiscale Chapman-Enskog expansion. Such a procedure shows that the LB model recovers the continuity equation and the viscous Saint-Venant (shallow water) equations. Discrepancies are third order in $\Delta t$ and $\Delta x$ and in the dissipative term. In recent literature [22], the viscous term is unfortunately incorrectly calculated. The correct form has been briefly indicated in an appendix of [5], omitting the complete expression for LB case. For this reason, we give below a full derivation of the hydrodynamic equations. We also give the explicit expression of the non-equilibrium part of the density distribution which, to the best of our knowledge, has never been published for the shallow water LB model. Note however that in our derivation we do not consider the case with the force term.

The multiscale Chapman-Enskog expansion method is described in detail in [2]. It contains several steps that are summarized below: 
(1) A Taylor expansion, up to second order of the LB dynamics (6)

$$
\Delta t \partial_{t} f_{i}+v_{i} \Delta t \partial_{x} f_{i}+\frac{1}{2} \Delta t^{2} \partial_{t}^{2} f_{i}+\frac{1}{2} v_{i}^{2} \Delta t^{2} \partial_{x}^{2} f_{i}+v_{i} \Delta t^{2} \partial_{x} \partial_{t} f_{i}=\frac{1}{\tau}\left(f_{i}^{e q}-f_{i}\right)
$$

(2) An expansion in a formal parameter $\epsilon$ (usually interpreted as the Knudsen number) of the distribution functions

$$
f_{i}=f_{i}^{e q}+\epsilon f_{i}^{(1)}+\epsilon^{2} f_{i}^{(2)}+\ldots
$$

(3) A multiscale analysis to separate the two time scales in the problem. Here we assume that the process is governed by a fast convective scale and a slow dissipative scale. Therefore we express the spatial and temporal variables $t$ and $x$ in terms of new variables $t_{1}, t_{2}$ and $x_{1}$.

$$
\begin{array}{r}
\partial_{t}=\epsilon \partial_{t_{1}}+\epsilon^{2} \partial_{t_{2}} \\
\partial_{x}=\epsilon \partial_{x_{1}}
\end{array}
$$

The multiscale approach gives a way to properly approximate the second order time derivative in (15).

(4) The first two moments of eq. (15) are taken (sum over $i$ and multiplication by $v_{i}$ and sum over $i$ ). The right-hand side exactly vanishes at all order in $\epsilon$, due to the conservation laws. We obtain, for the convective scale

$$
\begin{aligned}
\partial_{t_{1}} h+\partial_{x_{1}}(h u) & =0 \\
\partial_{t_{1}}(h u)+\partial_{x_{1}} \Pi^{e q} & =0
\end{aligned}
$$

and, for the dissipative scale

$$
\begin{aligned}
\partial_{t_{2}} h+\frac{1}{2} \Delta t \partial_{t_{1}}^{2} h+\frac{1}{2} \Delta t \partial_{x_{1}}^{2} \Pi^{e q}+\Delta t \partial_{x_{1}} \partial_{t_{1}}(h u) & =0 \\
\partial_{t_{2}}(h u)+\partial_{x_{1}} \Pi^{(1)}+\frac{1}{2} \Delta t \partial_{t_{1}}^{2}(h u)+\frac{1}{2} \Delta t \partial_{x_{1}}^{2} S^{e q}+\Delta t \partial_{x_{1}} \partial_{t_{1}} \Pi^{e q} & =0
\end{aligned}
$$

Then, after some algebra (see [2]) the two scales can be recombined to give the hydrodynamic equations, at the scale $x$ and $t$

$$
\begin{aligned}
\partial_{t} h+\partial_{x}(h u) & =0 \\
\partial_{t}(h u)+\partial_{x}\left[\Pi^{e q}+\epsilon \Pi^{(1)}+\frac{1}{2} \Delta t\left(\epsilon \partial_{t_{1}} \Pi^{e q}+\partial_{x} S^{e q}\right)\right] & =0
\end{aligned}
$$

where the quantities $\Pi$ and $S$ are tensors defined as

$$
\Pi=\sum_{i} v_{i}^{2} f_{i} \quad \Pi^{e q}=\sum_{i} v_{i}^{2} f_{i}^{e q} \quad \Pi^{(1)}=\sum_{i} v_{i}^{2} f_{i}^{(1)}
$$

and

$$
S=\sum_{i} v_{i}^{3} f_{i} \quad S^{e q}=\sum_{i} v_{i}^{3} f_{i}^{e q}
$$


We can recognize the continuity equation. But we see that eq. (24) is not yet in the final form of a shallow-water equation because $\Pi^{(1)}$ is unknown. To compute it we need to compute $f^{(1)}$.

(5) The nonequilibrium distribution $f^{(1)}$ can be obtained from the order $\mathcal{O}(\epsilon)$ of eq. (15)

$$
f_{i}^{(1)}=-\tau \Delta t\left[\partial_{t_{1}} f_{i}^{e q}+v_{i} \partial_{x_{1}} f_{i}^{e q}\right]
$$

The time derivative can be expressed as

$$
\partial_{t_{1}} f_{i}^{(0)}=\frac{\partial f_{i}^{(0)}}{\partial h} \partial_{t_{1}} h+\frac{\partial f_{i}^{(0)}}{\partial(h u)} \partial_{t_{1}}(h u)
$$

because, in the Chapman-Enskog expansion, we assume that $f$ depends on $x$ and $t$ only through the conserved quantities $h$ and $h u$. Then using equations (19) and (20), we obtain

$$
\epsilon f_{i}^{(1)}=-\tau \Delta t\left[-\frac{\partial f_{i}^{e q}}{\partial h} \partial_{x}(h u)-\frac{\partial f_{i}^{e q}}{\partial(h u)} \partial_{x} \Pi^{e q}+v_{i} \partial_{x} f_{i}^{e q}\right]
$$

Using that

$$
\partial_{x} \Pi^{e q}=2 u \partial_{x}(h u)+\left(g h-u^{2}\right) \partial_{x} h
$$

we obtain the explicit expressions:

$$
\begin{aligned}
\epsilon f_{0}^{(1)} & =\tau \Delta t\left[\left(1-\frac{g h}{v^{2}}-3 \frac{u^{2}}{v^{2}}\right) \partial_{x}(h u)+2\left(\frac{u^{2}}{v^{2}}-\frac{g h}{v^{2}}\right) u \partial_{x} h\right] \\
\epsilon f_{1}^{(1)} & =-\frac{1}{2} \epsilon f_{0}^{(1)} \\
\epsilon f_{2}^{(1)} & =-\frac{1}{2} \epsilon f_{0}^{(1)}
\end{aligned}
$$

Eqs. (30) are important because they give the relations between the standard hydrodynamic quantities and the nonequilibrium density distributions $\epsilon f_{i}^{(1)} \approx$ $f_{i}-f_{i}^{e q}$. We see that the $f^{(1)}$ 's depend on the spatial derivatives of $h$ and $u$. From these equations, an initial condition $f_{i}(x)=f_{i}^{e q}+\epsilon f_{i}^{(1)}$ can be built properly out of given values for $h(x), u(x), \partial_{x} h$ and $\partial_{x}(h u)$.

(6) Finally the dissipative contribution of (24)

$$
\Gamma=-\partial_{x}\left[\epsilon \Pi^{(1)}+\frac{1}{2} \Delta t \epsilon \partial_{t_{1}} \Pi^{e q}+\frac{1}{2} \Delta t \partial_{x} S^{e q}\right]
$$

can be computed from $f^{(1)}$. As above, we also replace $\epsilon \partial_{t_{1}} \Pi^{e q}$ by $\left(\partial \Pi^{e q} / \partial h\right) \partial_{t_{1}} h+$ $\left(\partial \Pi^{e q} / \partial h u\right) \partial_{t_{1}} h u$. After some algebra we obtain that

$$
\Gamma=\Delta t\left(\tau-\frac{1}{2}\right) \partial_{x}\left[\frac{-\partial \Pi^{e q}}{\partial h} \partial_{x} h u-\frac{\partial \Pi^{e q}}{\partial h u} \partial_{x} \Pi^{(0)}+\partial_{x} S^{e q}\right]
$$


Using the expressions

$$
\Pi^{e q}=\frac{1}{2} g h^{2}+h u^{2}=\frac{1}{2} g h^{2}+\frac{1}{h}(h u)^{2}
$$

and

$$
\partial_{x} S^{e q}=v^{2} \partial_{x} h u \quad h \partial_{x} u=\partial_{x} h u-u \partial_{x} h
$$

we obtain that

$$
\Gamma=\Delta t\left(\tau-\frac{1}{2}\right) \partial_{x}\left[\left(v^{2}-g h-3 u^{2}\right) \partial_{x} h u+2\left(u^{2}-g h\right) u \partial_{x} h\right]
$$

¿From the above expression for $\Gamma$, it follows that the shallow water equations with dissipation, resulting from the LB dynamics, are

$$
\partial_{t} h+\partial_{x} h u=0
$$

and

$$
\begin{aligned}
\partial_{t} h u+\partial_{x} h u^{2}+g \partial_{x} \frac{1}{2} h^{2}= & v^{2} \Delta t\left(\tau-\frac{1}{2}\right) \partial_{x}\left[\left(1-\frac{g h}{v^{2}}-3 \frac{u^{2}}{v^{2}}\right) \partial_{x} h u\right. \\
& \left.+2\left(\frac{u^{2}}{v^{2}}-\frac{g h}{v^{2}}\right) u \partial_{x} h\right]
\end{aligned}
$$

We observe that the shallow water equation associated with the LB model contains many contributions to the viscous terms, whose physical relevance remains to be discussed. However, we will see below that the 1D LB model remains numerically stable even when $\tau$ is close to $1 / 2$, provided the Froude number is not approaching 1 . Therefore, the viscous contributions can be made small and the LB model approximates the non-viscous shallow water equation.

Note also that in the limit of $h \rightarrow 0$ and $u \rightarrow 0$, we obtain the more standard viscous term

$$
\Gamma=v^{2} \Delta t\left(\tau-\frac{1}{2}\right) \partial_{x}^{2} h u
$$

However, it has to be noticed that even in this limit, the viscosity

$$
\nu_{0}=v^{2} \Delta t\left(\tau-\frac{1}{2}\right)
$$

is not at all the one pointed out in [22] which depends only on the lattice properties as $\Delta t c_{s}^{2}(\tau-1 / 2)$. Here, from (8) we have $c_{s}^{2}=v^{2} / 3$.

Our result (35) will be confirmed in section 4 in which the real part of the eigenvalues of the linearized model will be examined.

\section{Analysis of the steady state with zero flow}

In this section we derive an analytical solution of our discrete LB model when the external force term is present. To the best of our knowledge, exact solutions of an LB model with a non-constant force term have not been published before. 

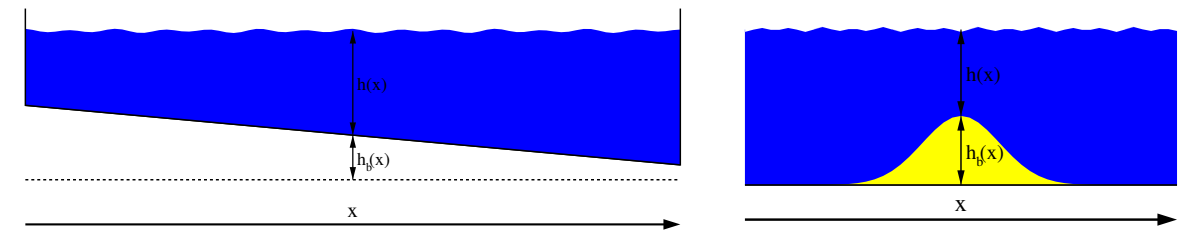

Figure 3: Two situations of a canal reach where $u=0$ and $h+h_{b}=$ const is an exact solution. Left: there is a net slope but two walls prevent flow motion. A computational domain with the so-called half-way bounce back left and right boundary conditions is used. Right: there is a local deformation of the bed but no global slope. A periodic computational domain can be used.

In order to solve the LB dynamics exactly we have to consider the simple situation sketched in Fig. 3. A fluid is at rest in a canal reach, with a free surface level which is horizontal. Therefore the solution to the problem is a water depth $h(x)$ such that $h(x)+h_{b}(x)=$ const for all $x$, where $h_{b}(x)$ is the bed height at location $x$. This simple benchmark gives a test of the accuracy of the way to add the external force to a LB model. We shall consider both Zhou's and Guo's methods. The result of this analysis is that Zhou's method solves the water profile to machine accuracy but no longer satisfies exactly the correct mass and momentum balances.

\subsection{Zhou's expression for the force term}

Zhou [22] proposed the following way to include the force term:

$$
\begin{aligned}
f_{0}(x, t+\Delta t) & =f_{0}(x, t)+\frac{1}{\tau}\left(f_{0}^{e q}-f_{0}\right) \\
f_{i}\left(x+v_{i} \Delta t, t+\Delta t\right) & =f_{i}(x, t)+\frac{1}{\tau}\left(f_{i}^{e q}-f_{i}\right)+w_{i} \frac{\Delta t}{c_{s}^{2}} v_{i} F_{i} \quad i \neq 0
\end{aligned}
$$

with

$$
F_{1}=F\left(x+\frac{\Delta x}{2}\right), \quad F_{2}=F\left(x-\frac{\Delta x}{2}\right), \quad w_{i} \frac{\Delta t}{c_{s}^{2}}=\frac{\Delta t}{2 v^{2}} .
$$

The water height, $h$, and water current, $h u$, are still given by

$$
h=\sum_{i} f_{i}=\sum_{i} f_{i}^{e q} \quad h u=\sum_{i} v_{i} f_{i}=\sum_{i} v_{i} f_{i}^{e q}
$$

despite the additional force term. As a consequence, we have

$$
\sum_{i} f^{n e q}=\sum_{i} v_{i} f_{i}^{n e q}=0
$$


For a fluid at rest, $u=0$, and we have the additional relations:

$$
f_{1}^{e q}=f_{2}^{e q} \equiv f^{e q}=\frac{1}{4 v^{2}} g h^{2}
$$

and

$$
f_{0}^{e q}=h-\frac{1}{2 v^{2}} g h^{2}
$$

Also, from the definition of $h u$, we have $0=\sum_{i} v_{i} f_{i}$ and thus $f_{1}=f_{2}$. In the steady state, the LB equations give

$$
f_{0}(x)=f_{0}(x)+\frac{1}{\tau}\left(f_{0}^{e q}-f_{0}\right) .
$$

Therefore

$$
f_{0}=f_{0}^{e q}=h-\frac{1}{2 v^{2}} g h^{2}
$$

This implies that

$$
f_{0}^{n e q}=f_{0}-f_{0}^{e q}=0
$$

and thus, from (38) $f_{1}^{n e q}+f_{2}^{n e q}=0$. Since for $u=0$ we also have $f_{1}^{n e q}=f_{2}^{n e q}$, we conclude that

$$
f_{1}^{n e q}=f_{2}^{n e q}=0
$$

Therefore, for the situation at rest, with Zhou's external force, the density distribution functions are simply

$$
f_{0}=f_{0}^{e q}=h-\frac{1}{2 v^{2}} g h^{2} \quad f_{1}=f_{2}=\frac{1}{4 v^{2}} g h^{2}
$$

With $f=f^{e q}$, the LB equation for $i=1$ reads

$$
f_{1}(x+\Delta x)=f_{1}(x)+\frac{\Delta t}{2 v} F\left(x+\frac{\Delta x}{2}\right)
$$

or, equivalently

$$
f_{1}(x+\Delta x / 2)=f_{1}(x-\Delta x / 2)+\frac{\Delta t}{2 v} F(x) .
$$

Using

$$
f_{1}=f_{2}=f^{e q}=\frac{1}{4 v^{2}} g h^{2},
$$

we obtain

$$
\frac{1}{4 v^{2}} g h^{2}\left(x+\frac{\Delta x}{2}\right)=\frac{1}{4 v^{2}} g h^{2}\left(x-\frac{\Delta x}{2}\right)+\frac{\Delta t}{2 v} F(x) .
$$

Therefore, Zhou's model imposes

$$
\frac{g}{2} \frac{h^{2}\left(x+\frac{\Delta x}{2}\right)-h^{2}\left(x-\frac{\Delta x}{2}\right)}{\Delta x}=F(x),
$$


which is a second-order accurate discrete form of the steady shallow-water eq. (2) with $u=0$

$$
\partial_{x} \frac{g}{2} h^{2}=F(x)
$$

The above two equations describe the situation of a fluid at rest in a container whose bottom is not flat. For the case we consider here, the force, $F$, depends on the derivative $\partial_{x} h_{b}(x)$ of the bed profile.

With $F=-g h \partial_{x} h_{b}(x)$ and $\partial_{x} \frac{g}{2} h^{2}=F(x)$ we clearly obtain $\partial_{x}\left(h+h_{b}\right)=0$, i.e. $h+h_{b}=$ const. In the discrete case, Zhou defines

$$
F\left(x+\frac{\Delta x}{2}\right)=-g \frac{h(x+\Delta x)+h(x)}{2}\left(\frac{h_{b}(x+\Delta x)-h_{b}(x)}{\Delta x}\right)
$$

Then, with $x_{-}=x-\Delta x / 2$ and $x_{+}=x+\Delta x / 2$ eq. (44) becomes:

$$
\frac{g}{2} \frac{h^{2}\left(x_{+}\right)-h^{2}\left(x_{-}\right)}{\Delta x}=-g \frac{h\left(x_{+}\right)+h\left(x_{-}\right)}{2}\left(\frac{h_{b}\left(x_{+}\right)-h_{b}\left(x_{-}\right)}{\Delta x}\right)
$$

Since

$$
h^{2}\left(x_{+}\right)-h^{2}\left(x_{-}\right)=\left[h\left(x_{+}\right)+h\left(x_{-}\right)\right]\left[h\left(x_{+}\right)-h\left(x_{-}\right)\right],
$$

the solution of this discrete equation is simply

$$
h\left(x_{+}\right)+h_{b}\left(x_{+}\right)=h\left(x_{-}\right)+h_{b}\left(x_{-}\right)=\text {const }
$$

Therefore, with Zhou's force, the water profile, in the steady state with $u=0$ is resolved correctly to machine accuracy.

\section{Mass and momentum balance equation}

However, in Zhou's model, the fact that $F$ is not calculated at the same point $x$ for $i=1$ and $i=2$, has the consequence that (by taking the first moment of eq. (37))

$$
h^{\text {out }}(x)=\sum_{i} f_{i}^{\text {out }}=\sum_{i} f_{i}^{\text {in }}+\frac{\Delta t}{2 v}\left(F\left(x+\frac{\Delta x}{2}\right)-F\left(x-\frac{\Delta x}{2}\right)\right) \neq h^{\text {in }}(x)
$$

and thus the mass is not exactly constant during the collision process. A Taylor expansion shows that this correction is second order in the lattice spacing, $\Delta x$,

$$
\frac{\Delta t}{2 v}\left(F\left(x+\frac{\Delta x}{2}\right)-F\left(x-\frac{\Delta x}{2}\right)\right)=F^{\prime}(x) \frac{(\Delta x)^{2}}{2 v^{2}}
$$

However, if we sum up $h^{\text {out }}(x)$ over all $x$, we recover exact mass conservation provided that $F\left(L+\frac{\Delta x}{2}\right)=F\left(-\frac{\Delta x}{2}\right)$. On the other hand, if for instance $h_{b}$ has a constant slope, there will be a net increase (or decrease) of mass during evolution. This effect is easily observed in numerical simulations in which $F(L+$ $\left.\frac{\Delta x}{2}\right) \neq F\left(-\frac{\Delta x}{2}\right)$. 
A similar analysis can be made for the momentum balance. By definition a force $F$ acting during a time $\Delta t$ will increase the momentum by an amount $\Delta j=F \Delta t$. Since the momentum is defined as $j=\sum_{i} f_{i} v_{i}$, we have

$$
F \Delta t=\sum_{i} f_{i}^{o u t} v_{i}-\sum_{i} f_{i}^{i n} v_{i}
$$

By multiplying (37) by $v_{i}$ and summing over $i$, we get (remember that, by construction of $\left.f^{e q}, \sum_{i} f_{i}^{e q} v_{i}=h u\right)$ :

$\Delta j=v\left(f_{1}^{\text {out }}-f_{2}^{\text {out }}\right)-v\left(f_{1}^{\text {in }}-f_{2}^{\text {in }}\right)=\frac{1}{\tau}\left(h u-\sum_{i} f_{i}^{\text {in }} v_{i}\right)+\frac{\Delta t}{2}(F(x+\Delta x / 2)+F(x-\Delta x / 2))$.

With the definition, $h u=j=\sum_{i} f_{i}^{i n} v_{i}$, we get the relation:

$$
\Delta j=\frac{\Delta t}{2}(F(x+\Delta x / 2)+F(x-\Delta x / 2))=\Delta t F+\Delta t \frac{(\Delta x)^{2}}{4} F^{\prime \prime} .
$$

Therefore, unless the second spatial derivative, $F^{\prime \prime}(x)$, vanishes, the momentum balance is only correct to first order in $\Delta x$.

\subsection{Guo's force model}

Guo's method [9] to add a body force is local and ensures exact mass conservation. Furthermore, the relation between the mometum, $\sum_{i} f_{i} v_{i}$, and the flow speed, $u$, is modified to impose an exact momentum balance.

In the case of a D1Q3 model, Guo's force model reads:

$$
\begin{aligned}
f_{i}^{\text {out }}(x, t) & =f_{i}(x, t)+\frac{1}{\tau}\left(f_{i}^{e q}-f_{i}\right)+\left(1-w_{i}\right) A+\frac{\Delta t}{c_{s}^{2}} v_{i} B \\
f_{i}\left(x+v_{i} \Delta t, t+\Delta t\right) & =f_{i}^{\text {out }}(x, t),
\end{aligned}
$$

where $A$ and $B$ have to be determined in terms of the actual body force applied to the system. Guo has shown that $A$ and $B$ also depend of the fluid speed, $u$.

The above formulation guarantees a local mass conservation since $\sum_{i} w_{i}=1$ and $\sum_{i} v_{i}=0$

$$
h^{\text {out }}=\sum_{i} f_{i}^{\text {out }}=\sum_{i} f_{i}+\frac{1}{\tau} \sum_{i}\left(f_{i}^{e q}-f_{i}\right)+A \sum_{i}\left(1-w_{i}\right)+\frac{\Delta t}{c_{s}^{2}} B \sum_{i} v_{i}=h^{\text {in }}
$$

In order to obtain an exact solution to the LB equation, let us ignore for a while Guo's results and let us assume that the $A$ and $B$ are still unknown. This will allow us to also discuss the case of an added force term as in (7).

We still define $j=\sum_{i} v_{i} f_{i}$ as the momentum. In case of a body force $F$, the change of momentum during a time $\Delta t$ is $F \Delta t$ and from the first moment of the LB eq. (48) we have:

$$
F \Delta t=\sum_{i} v_{i} f_{i}^{\text {out }}-\sum_{i} v_{i} f_{i}=\frac{1}{\tau}\left(\sum_{i} v_{i} f_{i}^{e q}-\sum_{i} v_{i} f_{i}\right)+\Delta t B .
$$


Therefore the momentum balance requires $\tau \Delta t F=h u-\sum_{i} v_{i} f_{i}+\tau \Delta t B$, because, by definition of $f^{e q}, \sum_{i} f_{i}^{e q} v_{i}=h u$. This leads to the following redefinition of the relation between the speed, $u$, and the momentum, $\sum_{i} v_{i} f_{i}$ :

$$
h u=\sum_{i} v_{i} f_{i}+\tau \Delta t(F-B) .
$$

Note at this stage that choosing $B=F$ would preserve the usual definition of $h u$ in terms of the first moment of the $f_{i}$. But, otherwise, this new relation may cause a new difficulty: from (3) we see that, in the shallow water model, $F$ may be a non-linear function of $u$. Therefore eq. (49) is an implicit definition of $u$.

Here we consider the case of a fluid at rest. When $u=0$ we have already observed that $f_{1}^{e q}=f_{2}^{e q}=f^{e q}=\frac{1}{4 v^{2}} g h^{2}$ and $f_{0}^{e q}=h-\frac{1}{2 v^{2}} g h^{2}$. In addition, from the new definition of $h u$, we have $0=\sum_{i} v_{i} f_{i}+\tau \Delta t(F-B)$ and thus

$$
f_{1}-f_{2}=-\tau \frac{\Delta t}{v}(F-B)
$$

Since $f_{1}^{e q}=f_{2}^{e q}$ the above relation also requires:

$$
f_{1}^{n e q}-f_{2}^{n e q}=-\tau \frac{\Delta t}{v}(F-B)
$$

In a time-independent-state, the LB equation for $i=0$ is:

$$
f_{0}(x)=f_{0}(x)+\frac{1}{\tau}\left(f_{0}^{e q}-f_{0}\right)-\frac{1}{3} A
$$

Therefore

$$
f_{0}=f_{0}^{e q}+\frac{\tau}{3} A \quad \text { i.e. } \quad f_{0}^{n e q}=\frac{\tau}{3} A
$$

For the other directions, $i=1$ or 2 , the LB equations yield

$$
f_{1}(x+\Delta x)=f_{1}(x)+\frac{1}{\tau}\left(f^{e q}-f_{1}\right)+\frac{5}{6} A+\frac{\Delta t}{2 v} B
$$

and

$$
f_{2}(x-\Delta x)=f_{2}(x)+\frac{1}{\tau}\left(f^{e q}-f_{2}\right)+\frac{5}{6} A-\frac{\Delta t}{2 v} B .
$$

By taking this last equation for $x+\Delta x$, we get

$$
f_{2}(x)=f_{2}(x+\Delta x)+\frac{1}{\tau}\left(f^{e q}(x+\Delta x)-f_{2}(x+\Delta x)\right)+\frac{5}{6} A(x+\Delta x)-\frac{\Delta t}{2 v} B(x+\Delta x)
$$

Adding eqs. (53) and (55), we get

$$
\left[f_{1}-f_{2}+\frac{1}{\tau} f_{2}^{n e q}-\frac{5}{6} A+\frac{\Delta t}{2 v} B\right]_{x+\Delta x}=\left[f_{1}-f_{2}-\frac{1}{\tau} f_{1}^{n e q}+\frac{5}{6} A+\frac{\Delta t}{2 v} B\right]_{x}
$$


From (50) and (51) we have $f_{1}-f_{2}=-\tau \frac{\Delta t}{v}(F-B)$ and $f_{1}^{n e q}=f_{2}^{n e q}-$ $\tau \frac{\Delta t}{v}(F-B)$. Therefore the above equation becomes

$$
\begin{array}{r}
{\left[-\tau \frac{\Delta t}{v}(F-B)+\frac{1}{\tau} f_{2}^{n e q}-\frac{5}{6} A+\frac{\Delta t}{2 v} B\right]_{x+\Delta x}=} \\
{\left[-\tau \frac{\Delta t}{v}(F-B)-\frac{1}{\tau} f_{2}^{n e q}+\frac{\Delta t}{v}(F-B)+\frac{5}{6} A+\frac{\Delta t}{2 v} B\right]_{x}}
\end{array}
$$

which we can finally write as

$$
\begin{array}{r}
{\left[-\tau \frac{\Delta t}{v} F+\frac{\Delta t}{v}\left(\tau+\frac{1}{2}\right) B+\frac{1}{\tau} f_{2}^{n e q}-\frac{5}{6} A\right]_{x+\Delta x}=} \\
{\left[(1-\tau) \frac{\Delta t}{v} F-\left(\frac{1}{2}-\tau\right) \frac{\Delta t}{v} B-\frac{1}{\tau} f_{2}^{n e q}+\frac{5}{6} A\right]_{x}}
\end{array}
$$

The structure of this equation is

$$
\left[G+\frac{1}{\tau} f_{2}^{n e q}\right]_{x+\Delta x}=\left[H-\frac{1}{\tau} f_{2}^{n e q}\right]_{x}
$$

with

$G=-\tau \frac{\Delta t}{v} F+\frac{\Delta t}{v}\left(\tau+\frac{1}{2}\right) B-\frac{5}{6} A \quad H=(1-\tau) \frac{\Delta t}{v} F-\left(\frac{1}{2}-\tau\right) \frac{\Delta t}{v} B+\frac{5}{6} A$

If we choose $A$ and $B$ such that $H(x)=-G(x)$, we obtain

$$
\left[G+\frac{1}{\tau} f_{2}^{n e q}\right]_{x+\Delta x}=-\left[G+\frac{1}{\tau} f_{2}^{n e q}\right]_{x}
$$

which implies that

$$
G+\frac{1}{\tau} f_{2}^{n e q}=0
$$

The condition that $H(x)=-G(x)$ requires:

$$
-\tau \frac{\Delta t}{v} F+\frac{\Delta t}{v}\left(\tau+\frac{1}{2}\right) B-\frac{5}{6} A=-(1-\tau) \frac{\Delta t}{v} F+\left(\frac{1}{2}-\tau\right) \frac{\Delta t}{v} B-\frac{5}{6} A
$$

and thus

$$
B=\left(1-\frac{1}{2 \tau}\right) F \quad \text { or } \quad F-B=\frac{1}{2 \tau} F
$$

This means that the definition (49) of $u$ in terms of the $f_{i}$ 's becomes

$$
h u=\sum_{i} v_{i} f_{i}+\frac{\Delta t}{2} F
$$

as previously obtained by Guo. Now, from (58) and $B=(1-1 /(2 \tau)) F$ we have

$$
\begin{aligned}
f_{2}^{n e q} & =-\tau G \\
& =\frac{\Delta t}{4 v} F+\frac{5 \tau}{6} A
\end{aligned}
$$


and, from (51)

$$
f_{1}^{n e q}=-\frac{\Delta t}{4 v} F+\frac{5 \tau}{6} A .
$$

In order to determine $A$ we use the condition $\sum_{i} f_{i}^{\text {neq }}=0$, resulting from the fact that $\sum_{i} f_{i}=h=\sum_{i} f_{i}^{e q}$. From (52), $f_{0}^{n e q}=(\tau / 3) A$,

$$
\frac{\tau}{3} A-\frac{\Delta t}{4 v} F+\frac{5 \tau}{3} A+\frac{\Delta t}{4 v} F=0
$$

and thus

$$
A=0
$$

Let us now compute the water profile $h(x)$. Eq. (53), with $A=0$ and $B=(1-1 /(2 \tau)) F$ is:

$$
f_{1}(x+\Delta x)=f_{1}(x)+\frac{\Delta t}{2 v} F .
$$

With $f_{1}=f_{1}^{e q}+f_{1}^{n e q}=\frac{1}{4 v^{2}} g h^{2}-\frac{\Delta t}{4 v} F$, this equation becomes

$$
\frac{1}{4 v^{2}} g h^{2}(x+\Delta x)=\frac{1}{4 v^{2}} g h^{2}(x)+\frac{\Delta t}{4 v}[F(x)+F(x+\Delta x)]
$$

that is

$$
g \frac{h(x+\Delta x)-h(x)}{\Delta x} \frac{h(x+\Delta x)+h(x)}{2}=\frac{1}{2}[F(x)+F(x+\Delta x)] .
$$

This relation has to be compared with the corresponding continuous equation $g h \partial_{x} h_{b}=F$.

For a non-flat profile $h_{b}(x)$ of the canal bed, we have $F=-g h \partial_{x} h_{b}$. In the discrete case, let us define

$$
F(x)=-g h(x) h_{b}^{\prime}(x)
$$

where $h_{b}^{\prime}$ is a discrete approximation of the $x$-derivative of $h_{b}$. Eq. (66) then becomes:

$$
\frac{h(x+\Delta x)-h(x)}{\Delta x}=-h_{b}^{\prime}(x)-h(x+\Delta x) \frac{h_{b}^{\prime}(x+\Delta x)-h_{b}^{\prime}(x)}{h(x+\Delta x)+h(x)} .
$$

If we take $h_{b}^{\prime}(x)=\left[h_{b}(x+\Delta x)-h_{b}(x)\right] / \Delta x$, we get

$$
h(x+\Delta x)+h_{b}(x+\Delta x)=h(x)+h_{b}(x)-\frac{h(x+\Delta x)}{h(x+\Delta x)+h(x)} h_{b}^{\prime \prime}(x)(\Delta x)^{2}
$$

where $h_{b}^{\prime \prime}(x)$ is defined as $h_{b}^{\prime \prime}(x)=\left[h_{b}^{\prime}(x+\Delta x)-h_{b}^{\prime}(x)\right] / \Delta x$. Therefore, unless $h_{b}^{\prime \prime}$ vanishes (which happens if the canal slope $h_{b}^{\prime}$ is constant), the exact solution $h+h_{b}=$ const with an error in $\mathcal{O}\left((\Delta x)^{2}\right)$. 
Therefore, Guo's force solves the problem with only first order accuracy. This contradicts the general idea that, in the LB model, the addition of a body force does not alter the second-order accuracy of the scheme.

Note that here, we have no boundary conditions that reduce the accuracy but we have used a first-order accurate approximation of $\partial_{x} h_{b}$. Therefore let us now consider a second order accurate version of $h_{b}^{\prime}$

$$
h_{b}^{\prime}(x)=\frac{h_{b}(x+\Delta x / 2)-h_{b}(x-\Delta x / 2)}{\Delta x}
$$

Note that we still have $h_{b}^{\prime}(x+\Delta x)-h_{b}^{\prime}(x) \propto \partial_{x}^{2} h_{b}(x+\Delta x / 2) \Delta x=\mathcal{O}(\Delta x)$

If we now define $\tilde{h}(x)=h(x+\Delta x / 2)$, eq. (67) becomes

$$
\tilde{h}(x+\Delta x / 2)+h_{b}(x+\Delta x / 2)=\tilde{h}(x-\Delta x / 2)+h_{b}(x-\Delta x / 2)+\mathcal{O}\left((\Delta x)^{2}\right)
$$

We again observe a departure from the constant profile of order $(\Delta x)^{2}$.

\subsection{The constant force model}

It is now interesting to investigate the constant force model as expressed in (7). In the formalism of this section, it corresponds to choosing

$$
B=F \quad A=0
$$

As a consequence, we obtain that, for $u=0, f_{1}^{n e q}=f_{2}^{n e q}$. With $f_{0}^{e q}=0$ (because $A=0$ ), we further have $f_{1}^{n e q}+f_{2}^{n e q}=0$. Thus $f_{1}^{n e q}=f_{2}^{n e q}=0$. Then eq. (57) becomes

$$
\left[\frac{1}{\tau} f_{2}^{n e q}+\frac{\Delta t}{2 v} F\right]_{x+\Delta x}=\left[-\frac{1}{\tau} f_{2}^{n e q}+\frac{\Delta t}{2 v} F\right]_{x}
$$

This no longer implies that both terms are zeros. Instead, the solution is

$$
f_{2}^{n e q}(x+\Delta x)=-f_{2}^{n e q}(x)-\tau \frac{\Delta t}{2 v}(F(x+\Delta x)-F(x))
$$

Unless $F=$ const, this contradicts the fact that $f_{2}^{n e q}=0$. Therefore, for $F=F(x)$, there is no steady state solution of the LB equation with $u=0$. This shows that the constant force model makes no sense when the force is not constant.

\section{The linearized model}

In this section we give an exact time-dependent solution of the linearized D1Q3 LB shallow water model. We consider the case of a periodic system, without external force, for which a discrete Fourier analysis can be done.

The spectrum of the evolution operator is investigated numerically for the full range of wave numbers $k$. In the hydrodynamic limit $k \rightarrow 0$ an exact 
expression for the eigenvalues can be found, accurate to $\mathcal{O}\left(k^{2}\right)$. The results of this section will confirm the validity of our Chapman-Enskog solution (35), demonstrate the second-order accuracy of the LB scheme and give the stability region of the linearized model.

\subsection{Linearisation of the shallow water equation}

We first derive the dispersion relation associated with eqs. $(34,35)$ linearized around a constant height, $h=h_{0}$, and constant speed, $u=u_{0}$. With $h_{0}+\Delta h$ and $u_{0}+\Delta u$, the linearized dissipative term (33) is

$\Gamma=v^{2} \Delta t\left(\tau-\frac{1}{2}\right) h_{0}\left[\left(1-\phi^{2}-3 \phi^{2} \operatorname{Fr}^{2}\right) \partial_{x}^{2} \Delta u+\left(1-3 \phi^{2}-\phi^{2} \operatorname{Fr}^{2}\right) \frac{u_{0}}{h_{0}} \partial_{x}^{2} \Delta h\right]$

where

$$
\phi=\frac{\sqrt{g h_{0}}}{v} \quad \operatorname{Fr}=\frac{u_{0}}{\sqrt{g h_{0}}}
$$

The quantitiy $\phi$ is the ratio of the wave speed to the lattice speed and Fr is the

Froude number. With these definitions we have that the water speed in lattice units is

$$
\frac{u_{0}}{v}=\operatorname{Fr} \phi
$$

We can now express the continuity and shallow water equation in a matrix form:

$$
\partial_{t}\left(\begin{array}{c}
\Delta h \\
\Delta u
\end{array}\right)=\left(\begin{array}{cc}
-u_{0} & -h_{0} \\
-g & -u_{0}
\end{array}\right) \partial_{x}\left(\begin{array}{c}
\Delta h \\
\Delta u
\end{array}\right)+\nu_{0}\left(\begin{array}{cc}
0 & 0 \\
r \frac{u_{0}}{h_{0}} & s
\end{array}\right) \partial_{x}^{2}\left(\begin{array}{c}
\Delta h \\
\Delta u
\end{array}\right)
$$

where

$$
\nu_{0}=\Delta t v^{2}\left(\tau-\frac{1}{2}\right)
$$

and

$$
r=1-3 \phi^{2}-\phi^{2} \operatorname{Fr}^{2} \quad \text { and } s=1-\phi^{2}-3 \phi^{2} \operatorname{Fr}^{2} .
$$

We now consider a solution of the form

$$
\left(\begin{array}{c}
\Delta h \\
\Delta u
\end{array}\right)=\exp (i \omega t+i k x)\left(\begin{array}{c}
\Delta h_{0} \\
\Delta u_{0}
\end{array}\right)
$$

and the matrix equation now reads

$$
\left[i \omega-i k\left(\begin{array}{cc}
-u_{0} & -h_{0} \\
-g & -u_{0}
\end{array}\right)+\nu_{0} k^{2}\left(\begin{array}{cc}
0 & 0 \\
r \frac{u_{0}}{h_{0}} & s
\end{array}\right)\right]\left(\begin{array}{c}
\Delta h_{0} \\
\Delta u_{0}
\end{array}\right)=0
$$

A solution exists only if

$$
\operatorname{det}\left(\begin{array}{cc}
i \omega+i k u_{0} & i k h_{0} \\
i k g+\nu_{0} k^{2} r \frac{u_{0}}{h_{0}} & i \omega+i k u_{0}+\nu_{0} s k^{2}
\end{array}\right)=0
$$


This yields an equation for $\omega$ whose solution is

$$
\omega_{ \pm}=\frac{1}{2}\left[-\left(2 u_{0} k-i \nu_{0} s k^{2}\right) \pm 2 k \sqrt{g h_{0}} \sqrt{1-i r \frac{\nu_{0} u_{0}}{g h_{0}} k-\frac{\nu_{0}^{2} s^{2}}{4 g h_{0}} k^{2}}\right]
$$

We are interested in a solution accurate to order $\mathcal{O}\left(k^{2}\right)$, which describes the hydrodynamical regime. Therefore we use the first-order Taylor expansion of $\sqrt{1+x}=1+x / 2$ and we obtain, using expressions (72) and (75) for $\phi, \mathrm{Fr}, r$ and $s$

$$
\omega_{ \pm}=\left(-u_{0} \pm \sqrt{g h_{0}}\right) k+i \frac{\nu_{0}}{2}\left[1-\phi^{2}-3 \phi^{2} \operatorname{Fr}^{2} \mp \operatorname{Fr}\left(1-3 \phi^{2}-\phi^{2} \operatorname{Fr}^{2}\right)\right] k^{2}
$$

which is the dispersion relation for the shallow water equation derived from the Chapman-Enskog expansion of the LB model. With $\Delta x=v \Delta t$, and $\nu_{0}$ given by (36) we can get a dimensionless form of this dispersion relation

$$
\begin{aligned}
\omega_{ \pm} \Delta t= & \left(-\frac{u_{0}}{v} \pm \sqrt{\frac{g h_{0}}{v^{2}}}\right)(k \Delta x) \\
& +\frac{i}{2}\left(\tau-\frac{1}{2}\right)\left[1-\phi^{2}-3 \phi^{2} \operatorname{Fr}^{2} \mp \operatorname{Fr}\left(1-3 \phi^{2}-\phi^{2} \operatorname{Fr}^{2}\right)\right](k \Delta x)^{2}
\end{aligned}
$$

In the next section, we shall compare this dispersion relation with the eigenval-

ues of the LB dynamics. For this purpose we shall need to know $e^{i \omega_{ \pm} \Delta t}$. Up to order $\mathcal{O}\left(k^{2}\right)$, we can write

$$
e^{i \omega_{ \pm} \Delta t}=1+i \beta_{ \pm} k \Delta x-\alpha_{ \pm}(k \Delta x)^{2}
$$

From (78) we get

$$
\beta_{ \pm}=-\frac{u_{0}}{v} \pm \sqrt{\frac{g h_{0}}{v^{2}}}=-\phi(\operatorname{Fr} \mp 1)
$$

and $\alpha_{ \pm}$

$$
\alpha_{ \pm}=\frac{1}{2}\left(\tau-\frac{1}{2}\right)\left[1-\phi^{2}\left(1+3 \operatorname{Fr}^{2}\right) \pm\left(\phi^{2}\left(3+\operatorname{Fr}^{2}\right)-1\right) \operatorname{Fr}\right]+\frac{1}{2} \beta^{2}
$$

Relation (79) will be re-obtained in the next section by an exact solution of the eigenvalue problem accurate to order $\mathcal{O}\left(k^{2}\right)$. See eq. (95).

\subsection{Linearization of the LB equations}

Let us now consider the linearization of the LB dynamics around $h=h_{0}$ and $u=u_{0}$. If $h_{0}$ and $u_{0}$ are constant $f_{i}=f_{i}^{e q}\left(h_{0}, u_{0}\right)$ is a solution of the LB 
equations (6) with $f^{e q}$ given by (13). We now consider a small perturbation $\epsilon_{i}$ around $f_{i}^{e q}\left(h_{0}, u_{0}\right)$

$$
f_{i}=f_{i}^{e q}\left(h_{0}, u_{0}\right)+\epsilon_{i}
$$

Then we immediatly obtain

$$
\begin{gathered}
h=\sum_{i} f_{i}=h_{0}+\sum \epsilon_{i}, \quad h u=\sum_{i} f_{i} v_{i}=h_{0} u_{0}+\left(\epsilon_{1}-\epsilon_{2}\right) v \\
u=\frac{h u}{h}=u_{0}-\frac{u_{0}}{h_{0}} \sum_{i} \epsilon_{i}+\left(\epsilon_{1}-\epsilon_{2}\right) \frac{v}{h_{0}} \\
h u^{2}=h_{0} u_{0}^{2}-u_{0}^{2} \sum_{i} \epsilon_{i}+2\left(\epsilon_{1}-\epsilon_{2}\right) v u_{0}
\end{gathered}
$$

We can now compute $f_{i}^{e q}(h, u)$ for all $i$.

$$
\begin{gathered}
f_{0}^{e q}(h, u)=f_{0}^{e q}\left(h_{0}, u_{0}\right)+\left(1-\frac{g h_{0}}{v^{2}}+\frac{u_{0}^{2}}{v^{2}}\right) \sum_{i} \epsilon_{i}-\frac{2 u_{0}}{v}\left(\epsilon_{1}-\epsilon_{2}\right) \\
f_{1}^{e q}(h, u)=f_{1}^{e q}\left(h_{0}, u_{0}\right)+\frac{1}{2}\left(\frac{g h_{0}}{v^{2}}-\frac{u_{0}^{2}}{v^{2}}\right) \sum_{i} \epsilon_{i}+\left(\frac{1}{2}+\frac{u_{0}}{v}\right)\left(\epsilon_{1}-\epsilon_{2}\right)(84) \\
f_{2}^{e q}(h, u)=f_{2}^{e q}\left(h_{0}, u_{0}\right)+\frac{1}{2}\left(\frac{g h_{0}}{v^{2}}-\frac{u_{0}^{2}}{v^{2}}\right) \sum_{i} \epsilon_{i}-\left(\frac{1}{2}-\frac{u_{0}}{v}\right)\left(\epsilon_{1}-\epsilon_{2}\right)(85)
\end{gathered}
$$

Then, in terms of the perturbation, $\epsilon_{i}$, the LB shallow water equation becomes

$$
\left(\begin{array}{c}
\epsilon_{0}(x, t+\Delta t) \\
\epsilon_{1}(x+v \Delta t, t+\Delta t) \\
\epsilon_{2}(x-v \Delta t, t+\Delta t)
\end{array}\right)=M\left(\begin{array}{c}
\epsilon_{0}(x, t) \\
\epsilon_{1}(x, t) \\
\epsilon_{2}(x, t)
\end{array}\right)
$$

with

$M=\frac{1}{\tau}\left(\begin{array}{ccc}\tau-\phi^{2}\left(1-\mathrm{Fr}^{2}\right) & 1-\phi^{2}\left(1-\mathrm{Fr}^{2}\right)-2 \frac{u_{0}}{v} & 1-\phi^{2}\left(1-\mathrm{Fr}^{2}\right)+2 \frac{u_{0}}{v} \\ \frac{\phi^{2}}{2}\left(1-\mathrm{Fr}^{2}\right) & \tau-\frac{1}{2}+\frac{\phi^{2}}{2}\left(1-\mathrm{Fr}^{2}\right)+\frac{u_{0}}{v} & \frac{\phi^{2}}{2}\left(1-\mathrm{Fr}^{2}\right)-\frac{1}{2}-\frac{u_{0}}{v} \\ \frac{\phi^{2}}{2}\left(1-\mathrm{Fr}^{2}\right) & \frac{\phi^{2}}{2}\left(1-\mathrm{Fr}^{2}\right)-\frac{1}{2}+\frac{u_{0}}{v} & \tau-\frac{1}{2}+\frac{\phi^{2}}{2}\left(1-\mathrm{Fr}^{2}\right)-\frac{u_{0}}{v}\end{array}\right)$

where $\phi$ and $\mathrm{Fr}$ are defined in (72). Thus, $M$ depends on 3 dimensionless parameters, which are $\tau \geq 1 / 2, \phi^{2}$ and $\mathrm{Fr}^{2}$.

We can now analyze the LB scheme by taking the discrete Fourier transform of eq. (86). We define

$$
\epsilon(x, t)=\sum_{k} A_{k}(t) e^{i k x}
$$

where $k=2 \ell \pi /(N \Delta x), \quad \ell=0,1, \ldots,(N-1)$ and we obtain

$$
A_{k}(t+\Delta t)=M_{k}(\phi, \mathrm{Fr}, \tau) A_{k}(t)
$$


with

$$
M_{k}=\left(\begin{array}{ccc}
1 & 0 & 0 \\
0 & e^{-i k \Delta x} & 0 \\
0 & 0 & e^{i k \Delta x}
\end{array}\right) M
$$

To simplify the notation, we define

$$
a=\frac{\phi^{2}\left(1-\mathrm{Fr}^{2}\right)}{\tau} \quad b=\frac{1}{\tau} \frac{u_{0}}{v}
$$

and

$$
c=-\frac{1}{2 \tau}+\frac{a}{2}+b \quad d=-\frac{1}{2 \tau}+\frac{a}{2}-b
$$

Then, the matrix $M_{k}$ reads

$$
M_{k}=\left(\begin{array}{ccc}
1-a & -2 c & -2 d \\
\frac{a}{2} e^{-i k \Delta x} & (1+c) e^{-i k \Delta x} & d e^{-i k \Delta x} \\
\frac{a}{2} e^{i k \Delta x} & c e^{i k \Delta x} & (1+d) e^{i k \Delta x}
\end{array}\right)
$$

Let us now show how the eigen values of $M_{k}$ can be related to the dispersion relation found in (77). We consider solutions of the form $\epsilon(x, t) \propto e^{i k x+i \omega t}$ as we did for the linearized shallow water equation. Here, it means that we assume that $A_{k}(t)$ can be written as $e^{i \omega t} A_{k}(0)$. Eq. (88) then reads

$$
e^{i \omega t} e^{i \omega \Delta t} A_{k}(0)=M_{k} e^{i \omega t} A_{k}(0) .
$$

A solution exists provided that

$$
\left(M_{k}-e^{i \omega \Delta t} I\right) A_{k}(0)=0
$$

has non-trivial solutions. That means that $e^{i \omega \Delta t}$ must be an eigen value of $M_{k}$. Eq. (79) gives the expected expression of $e^{i \omega \Delta t}$, in the hydrodynamic limit, to order $\mathcal{O}\left(k^{2}\right)$.

The eigenvalues of $M_{k}$ can easily be found numerically for all values of $\tau$, $\phi, \mathrm{Fr}$ and $k$. This is discussed in the next section. But before, we consider an analytical solution of the eigenvalue problem, accurate to order $\mathcal{O}\left(k^{2}\right)$. With

$$
\gamma_{-}=e^{-i k \Delta x} \quad \gamma_{+}=e^{i k \Delta x}
$$

the eigenvalue equation associated with $M_{k}$ reads:

$$
\begin{aligned}
(1-\lambda)\left(\gamma_{-}-\lambda\right)\left(\gamma_{+}-\lambda\right) & +c \gamma_{-}\left(\gamma_{+}-\lambda\right)(1-\lambda) \\
& +d \gamma_{+}\left(\gamma_{-}-\lambda\right)(1-\lambda) \\
& -a\left(\gamma_{-}-\lambda\right)\left(\gamma_{+}-\lambda\right)=0
\end{aligned}
$$

For $\gamma_{-}=\gamma_{+}=1$ i.e. for a wave number $k=0$, the above equation reads

$$
(1-\lambda)^{2}(1+c+d-a-\lambda)=0
$$


Since $c+d-a=-\frac{1}{\tau}$ the three eigenvalues are

$$
\lambda_{+}=1 \quad \lambda_{-}=1 \quad \lambda_{3}=1-\frac{1}{\tau}
$$

When the Froude number is $1, a=0$ and (91) becomes

$$
(1-\lambda)\left[\left(\gamma_{-}-\lambda\right)\left(\gamma_{+}-\lambda\right)+c \gamma_{-}\left(\gamma_{+}-\lambda\right)+d \gamma_{+}\left(\gamma_{-}-\lambda\right)\right]=0
$$

There is clearly an eigenvalue $\lambda=1$ and the other two are defined through

$$
\lambda^{2}-\lambda\left[\gamma_{+}+\gamma_{-}+c \gamma_{-}+d \gamma_{+}\right]+1+c+d=0
$$

In general, eq. (91) can be written as

$$
\lambda^{3}-\lambda^{2}\left(1-a+\gamma_{+}+\gamma_{-}+c \gamma_{-}+d \gamma_{+}\right)+\lambda\left[1+c+d+\left(\gamma_{+}+\gamma_{-}\right)(1-a)+c \gamma_{-}+d \gamma_{+}\right]+\frac{1}{\tau}-1=0
$$

The two eigenvalues $\lambda_{ \pm}$corresponding to water height $h$ and water flow $h u$ are such that $\lambda_{ \pm}=1$, for $k=0$, due to the conservation laws. Therefore, to order $\mathcal{O}\left(k^{2}\right)$, we have:

$$
\lambda=1+i \beta k \Delta x-\alpha(k \Delta x)^{2} \quad \gamma_{+}=1+i k \Delta x-\frac{1}{2}(k \Delta x)^{2} \quad \gamma_{-}=1-i k \Delta x-\frac{1}{2}(k \Delta x)^{2}
$$

where $\alpha$ and $\beta$ are parameters to be determined. Note that we could similarly obtain the third eigenvalue $\lambda_{3}$ of the problem because, for $k=0$, eq. (92) tells us that this eigenvalue is $1-(1 / \tau)$.

Eq. (91) can then be solved order by order. As this is a rather tedious and lengthy (but straigtforward) calculation, we only give the results.

Order $\mathcal{O}\left(k^{2}\right)$, value of $\beta$ : At order $\mathcal{O}\left(k^{2}\right)$, eq. (91) yields

$$
\beta_{ \pm}=-\phi(\operatorname{Fr} \mp 1)=-\frac{u_{0}}{v} \pm \sqrt{\frac{g h_{0}}{v^{2}}}
$$

as expected from the dispersion relation describing waves propagating at speeds $-u_{0} \pm \sqrt{g h_{0}}$.

Order $\mathcal{O}\left(k^{3}\right)$, value of $\alpha$ : At order $\mathcal{O}\left(k^{3}\right)$, we obtain a condition for $\alpha$ which is

$$
\alpha_{ \pm}=\frac{1}{2}\left(\tau-\frac{1}{2}\right)\left[1-\phi^{2}\left(1+3 \mathrm{Fr}^{2}\right) \pm\left(\phi^{2}\left(\mathrm{Fr}^{2}+3\right)-1\right) \mathrm{Fr}\right]+\frac{1}{2} \beta^{2}
$$

Relations (94) and (95) are in perfect agreement with the dispersion relation obtained from the Chapman-Enskog expansion of the LB shallow water model. See eqs. (80) and (81). 


\subsection{Numerical analysis of the eigenvalue problem}

In this section we compare the eigenvalues $\lambda$ of $M_{k}$ obtained numerically (Matlab solutions) for all values of $0 \leq k \Delta x<2 \pi$ and our analytical expression $\lambda_{ \pm}=$ $e^{i \omega_{ \pm} \Delta t}$ with $i \omega_{ \pm} \Delta t$ given by (78), namely

$$
\begin{aligned}
i \omega_{ \pm} \Delta t= & i\left(-\frac{u_{0}}{v} \pm \sqrt{\frac{g h_{0}}{v^{2}}}\right)(k \Delta x) \\
& -\frac{1}{2}\left(\tau-\frac{1}{2}\right)\left[1-\phi^{2}-3 \phi^{2} \operatorname{Fr}^{2} \mp \operatorname{Fr}\left(1-3 \phi^{2}-\phi^{2} \mathrm{Fr}^{2}\right)\right](k \Delta x)^{2}
\end{aligned}
$$

In Fig. 4, we show $\operatorname{Re}\left(\lambda_{ \pm}\right)$and $\operatorname{Im}\left(\lambda_{ \pm}\right)$for two arbitrary choices of the model parameters $\phi$, Fr and $\tau$. The solid lines correspond to our analytical expression $e^{i \omega_{ \pm} \Delta t}$ and the dots to the exact eigenvalue (found numerically). For Froude number $\mathrm{Fr}=1$, we observe in the lower right panel that the eigenvalue has a null imaginary part due to the fact that the wave is at rest. For small enough wave number $k$, we clearly observe an agreement between the eigenvalues of the LB model and the dispersion relation of the shallow water equation. Actually, Fig. 5 shows the quantities

$$
\Delta(k)=\left|\operatorname{Re}\left(\lambda-e^{i \omega_{ \pm} \Delta t}\right)\right| \quad \text { or } \quad \Delta(k)=\left|\operatorname{Im}\left(\lambda-e^{i \omega_{ \pm} \Delta t}\right)\right|
$$

as a function of the wave number $k$. The quantity $\Delta$ is the difference between the shallow water dispersion equation and the LB model. We see that the LB model is second-order accurate because the error $\Delta$ grows as $\mathcal{O}\left((k \Delta x)^{3}\right)$ for the imaginary part (i.e. the wave propagation process) and grows as $\mathcal{O}\left((k \Delta x)^{4}\right)$ for the real part (i.e. the dissipation process). The results of this section confirm the validity of our analytical derivation (Chapman-Enskog expansion) and confirm that the LB model is a second order accurate solver of the shallow water eq. (35).

\subsection{Numerical stability}

The stability of the LB scheme is guaranteed if $|\lambda|$, the norm of the eigenvalues of $M_{k}$, is not larger than 1 for all $k=2 \ell \pi /(N \Delta x)$, with $\ell=0,1, \ldots,(N-1)$. Our analytical expression (79) approximates the value of $\lambda$ only for small-tomoderate values of $k$. Therefore this expression cannot be used to assess the parameter range for which unconditional stability is achieved. On the other hand, a numerical investigation can be considered. We simply have to explore the space of possible values for the parameters $\phi, \operatorname{Fr}$ and $\tau$. For each of them one computes all eigenvalues $\lambda$ of $M_{k}(\tau, \phi, \mathrm{Fr})$. If, for all $k,|\lambda| \leq 1$, then the LB model is unconditionally stable for the chosen set of parameters.

As an illustration, Fig. 6 shows the norm of the three eigenvalues of $M_{k}(\tau, \phi, \mathrm{Fr})$, as a function of all values of the wave numbers $0 \leq k \Delta x<2 \pi$. We observe a numerical instability of the model for the chosen parameters because some eigenvalue have a norm larger than one. A systematic investigation of the numerical 

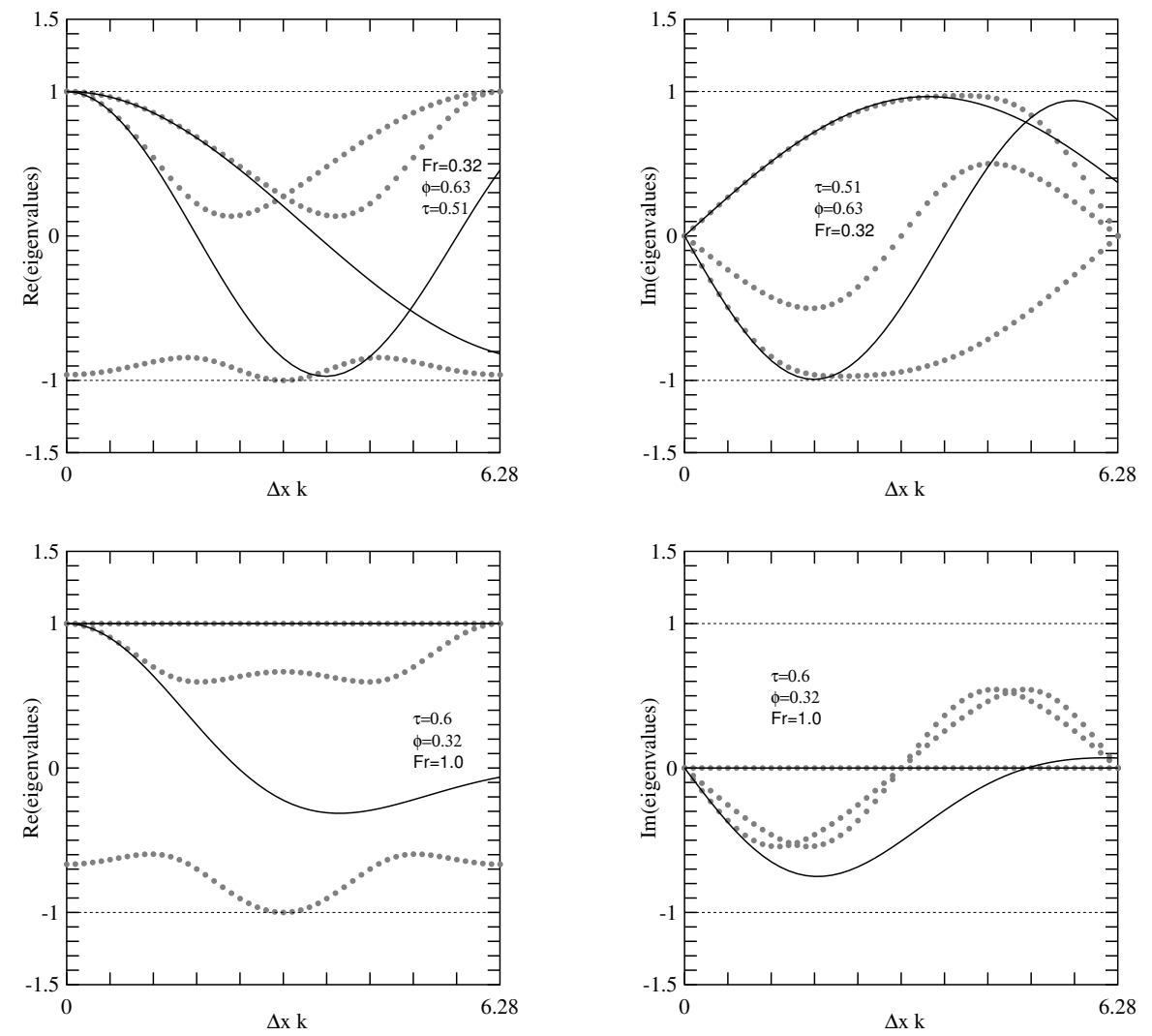

Figure 4: The real (left) and imaginary (right) parts of the eigenvalues $\lambda$ of $M_{k}(\tau, \phi, F r)$, as a function of $k \Delta x$ The eigenvalues found numerically are shown as dots and are compared to the shallow water dispersion relation $e^{i \omega_{ \pm} \Delta t}$ (solid lines). This figure illustrates two possible choices of the model parameters $\tau, \phi$ and Fr. 

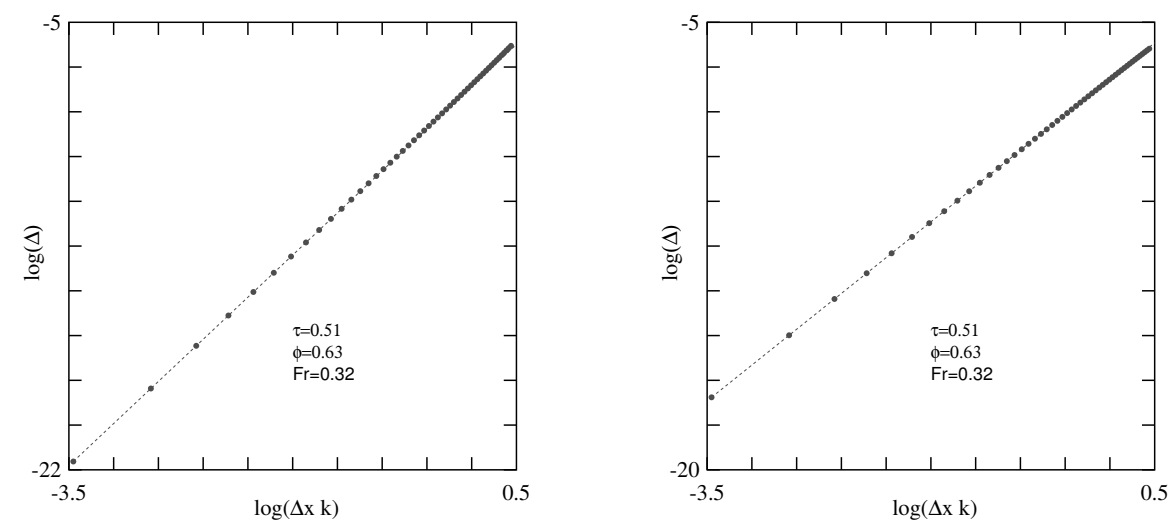

Figure 5: The dots show the value of $\Delta(k \Delta x)$, as given by (97) in a log-log plot. On the left, the accuracy of the real part of the dispersion relation is found to be second order because $\Delta(k \Delta x) \propto k^{4}$, as indicated by the solid line of slope 4. On the right, we show the accuracy of the imaginary part of the dispersion relation. The error between the LB model and the shallow water equation grows as $(k \Delta x)^{3}$, as proved by the solid line which has slope 3 .
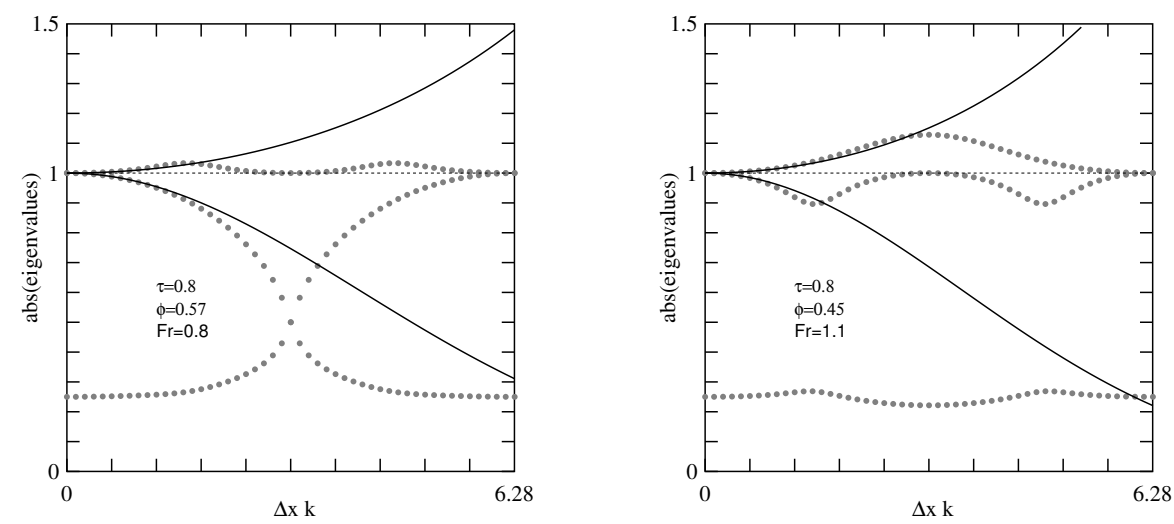

Figure 6: Norm of the eigenvalues of $M_{k}(\tau, \phi, \mathrm{Fr})$, as a function of $k \Delta x$, outside the numerical stability region. Left and right panels illustrate two different choices of $\tau, \phi$ and Fr. The dots show the three eigenvalues obtained numerically and the lines corresponds to $e^{i \omega_{ \pm} \Delta t}$. 


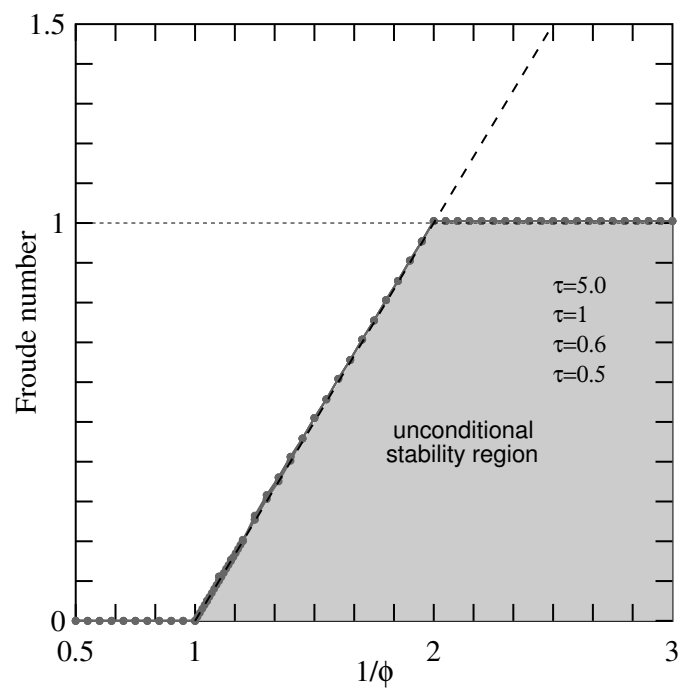

Figure 7: Stability region in the $\phi^{-1}-$ Fr plane, for different values of $\tau$ (gray dots), which all give the same limit. The black dashed line is the theoretical value corresponding to the Courant condition (99).

stability is given in Fig. 7. We observe that the relaxation time, $\tau$, plays no role in the stability of the D1Q3 model for the shallow water, provided it is larger than or equal to $1 / 2$.

We note that Froude number, Fr $=1$, is possible, provided that $\phi$ is small enough (i.e $1 / \phi$ large enough).

The stability range observed in Fig. 7 can be explained by a simple theoretical argument. Since the LB model describes waves propagating at speed $-u_{0} \pm \sqrt{g h_{0}}$, we must have the conditions

$$
-u_{0}+\sqrt{g h_{0}}<v \quad-u_{0}-\sqrt{g h_{0}}>-v
$$

so that the waves propagate slower than the lattice speed $v$, the speed at which information travels in the lattice. This is a kind of a Courant condition. By dividing these equations by $\sqrt{g h_{0}}$, we obtain

$$
1-\frac{1}{\phi}<\operatorname{Fr}<\frac{1}{\phi}-1
$$

This line is represented in Fig. 7 (solid, dashed line) and shows a very good agreement with the numerically observed stability limit.

We can also explain these limits of the stability region by analyzing the dispersion relation (78). We can write $e^{i \omega_{ \pm} \Delta t}=e^{i \beta_{ \pm} k \Delta x} e^{-\eta_{ \pm} k^{2}(\Delta x)^{2}}$ where

$$
\eta_{ \pm}=\frac{1}{2}\left(\tau-\frac{1}{2}\right)\left[1-\phi^{2}-3 \phi^{2} \operatorname{Fr}^{2} \mp \operatorname{Fr}\left(1-3 \phi^{2}-\phi^{2} \operatorname{Fr}^{2}\right)\right]
$$


Clearly, $\eta<0$ is a sufficient condition for the numerical scheme to be unstable, because, for $k \rightarrow 0, e^{i \omega_{ \pm} \Delta t} \rightarrow \lambda_{ \pm}$. When $\tau \geq 1 / 2$, a negative value of $\eta$ depends only on the choice of $\phi$ and Fr. It turns out that $\eta_{+}$can be factorized as

$$
\eta_{+}=-\frac{1}{2}\left(\tau-\frac{1}{2}\right) \phi^{2}(\mathrm{Fr}+1)\left(\mathrm{Fr}-\frac{1}{\phi}+1\right)\left(\mathrm{Fr}+\frac{1}{\phi}+1\right)
$$

Thus, for $\phi \geq 0$ and $\mathrm{Fr} \geq 0, \eta_{+}$becomes negative if

$$
\operatorname{Fr}-\frac{1}{\phi}+1>0 \quad \text { i.e. } \quad \operatorname{Fr}>\frac{1}{\phi}-1
$$

This is precisely the limit we found from the Courant condition.

Furthermore, we see that $\eta_{-}$can be factorized as

$$
\eta_{-}=\frac{1}{2}\left(\tau-\frac{1}{2}\right) \phi^{2}(\mathrm{Fr}-1)\left(\mathrm{Fr}-1-\frac{1}{\phi}\right)\left(\mathrm{Fr}-1+\frac{1}{\phi}\right)
$$

In the region obeying the Courant condition (i.e. $\operatorname{Fr}<\phi^{-1}-1$ ) we certainly have Fr $<\phi^{-1}+1$ and $\mathrm{Fr}>1-\phi^{-1}$ Therefore the condition for $\eta_{-}$to be negative (and the numerical scheme unstable) is $\mathrm{Fr} \geq 1$, as already obtained numerically.

These limits are also verified by the simulation in section 5.5

\section{Benchmark}

In this section, we validate numerically the analytical description derived in the previous section. More specifically we compare the LB model with the Preissmann scheme and the finite volume (FV) method with respect to the numerical stability, precision and performance.

\subsection{Simulation setup}

We consider the steady flow in a canal of length $L$ where the inflow discharge and the outflow water height are fixed at values $Q_{0}$ and $h_{0}$. In this case, the analytical solution can be obtained by integrating the ordinary differential equation for $h(x)$ :

$$
\partial_{x} h=\frac{g h(I-J)}{g h-u^{2}}
$$

with the boundary condition $h(L)=h_{0}$ and $J=\frac{n^{2} Q_{0}^{2}}{B^{2} h^{2}\left(\frac{B h}{B+2 h}\right)^{4 / 3}}, u=\frac{Q_{0}}{B h}$.

This exact solution is calculated by using the ode 45 solver of Matlab, and referenced as $h_{r e f}$. This benchmark is illustrated in Fig. 8.

The numerical schemes we want to compare are time-dependent solvers. We start the simulation with an initial condition and let the fluid reach its new steady state. The initial condition is a uniform profile of the water height 

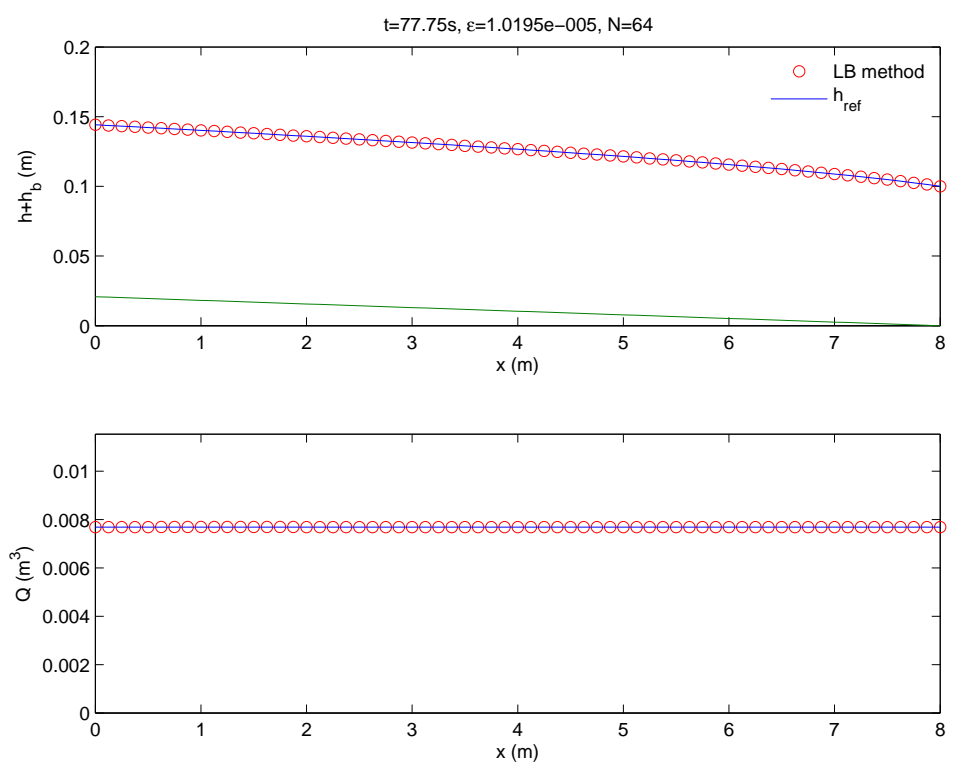

Figure 8: Steady state water level (above) and discharge (below) used for the comparison benchmark. The continuous line represents the reference solution computed from a numerical integration of the steady state shallow water equation.

$h(x, 0)=h_{0}$ and discharge $Q(x, 0)=Q_{e}$. Where $Q_{e}$ is the discharge corresponding to this uniform water height and calculated by the condition $I=J$. So, $Q_{e}=\frac{\sqrt{I} B h_{0}}{n}\left(\frac{B h}{B+2 h}\right)^{2 / 3}$.

In order to avoid a sudden change, the inflow discharge, $Q_{i n}$, will be increased in ramp from the initial value $Q_{e}$ to the final value $Q_{0}$. For the numerical application, we get $h_{0}=0.1 \mathrm{~m}, Q_{0}=1.5 Q_{e}, I=2,6.10^{-3}, B=0.1 \mathrm{~m}$ and $n=0.0103$.

All three methods are implemented in Matlab and none of them has been fine tuned for performance optimization. The FV code was initially developed by Guy Simpson [19].

\subsection{Preissmann implicit scheme}

The approximation of the function, $f(h$ or $u$ ), and its derivatives in space and in time is based on the following expression:

$$
\begin{aligned}
& f(x, t)=(1-\theta)\left[\phi f_{i+1}^{j}+(1-\phi) f_{i}^{j}\right]+\theta\left[\phi f_{i+1}^{j+1}+(1-\phi) f_{i}^{j+1}\right] \\
& \frac{\partial f}{\partial x}(x, t)=\frac{1}{\Delta x}\left[(1-\theta)\left(f_{i+1}^{j}-f_{i}^{j}\right)+\theta\left(f_{i+1}^{j+1}-f_{i}^{j+1}\right)\right] \\
& \frac{\partial f}{\partial t}(x, t)=\frac{1}{\Delta t}\left[(1-\phi)\left(f_{i}^{j+1}-f_{i}^{j}\right)+\phi\left(f_{i+1}^{j+1}-f_{i+1}^{j}\right)\right]
\end{aligned}
$$




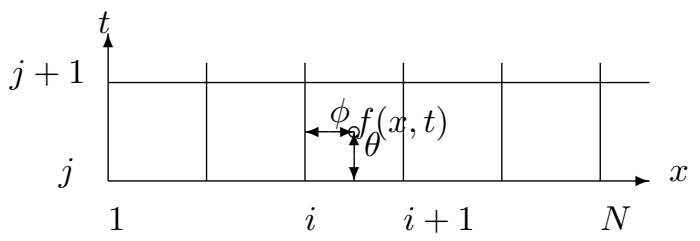

Figure 9: Preissmann implicit scheme

where $i$ is the space index, $j$ the time index and $0 \leq \theta \leq 1,0 \leq \phi \leq 1$, are weighting coefficients. If $\theta>0.5$ we get an unconditionally stable scheme. For simulation, we choose $\theta=0.75$ and $\phi=0.5$.

To solve the shallow water equation, we perform the Preissmann scheme for $h$ and $u$ in time and space, and obtain a system of nonlinear equations that can be solved by using the Newton-Raphson method. The boundary condition can be carried out by adding two equations: $B h_{1} u_{1}=Q_{i n}$ and $h_{N}=h_{0}$.

\section{$5.3 \quad$ Finite volume method}

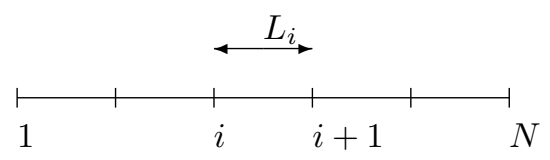

Figure 10: Finite volume method

Firstly, we rewrite the shallow-water equations as:

$$
\frac{\partial \mathbf{U}}{\partial t}+\frac{\partial \mathbf{E}}{\partial x}=\mathbf{S}
$$

where $\mathbf{U}=\left[\begin{array}{c}h \\ h u\end{array}\right]$ is the solution vector, $\mathbf{E}=\left[\begin{array}{c}h u \\ \frac{1}{2} g h^{2}+h u^{2}\end{array}\right]$ is the flux vector, and $\mathbf{S}=\left[\begin{array}{c}0 \\ g h(I-J)\end{array}\right]$ is the source vector.

By integrating (105) over an arbitrary segment $L_{i}$, the basic equation of the finite volume method is obtained:

$$
\frac{\partial}{\partial t} \int_{L_{i}} \mathbf{U} d x+[\mathbf{E}]_{x_{i}}^{x_{i+1}}=\int_{L_{i}} \mathbf{S} d x
$$

Rewriting the last equation in discrete form, the governing equations become:

$$
L_{i} \frac{\Delta \mathbf{U}_{i}}{\Delta t}+[\mathbf{E}]_{x_{i}}^{x_{i+1}}=L_{i} \mathbf{S}_{i} .
$$

The flux can be estimated by solving a series of local Riemann problems (See [19].), (107) can now be solved by the standard explicit forward Euler method. Note that in the code which was provided to us the Riemann problem is solved 
with first order accuracy, although a second order accurate solution is also possible.

The boundary condition for the inflow discharge is carried out by setting $h_{1}=h_{2}$ and $B h_{1} u_{1}=Q_{i n}$; the boundary condition for the outflow water height is implemented as $h_{N}=h_{0}$ and $h_{N} u_{N}=h_{N-1} u_{N-1}$.

\subsection{LB method}

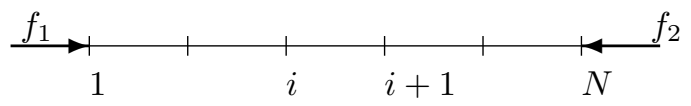

Figure 11: LB method

The LB scheme has been described in full detail the previous sections. Here we only describe how to implement the boundary conditions. In reference to Fig. 11 the inflow discharge is obtained by imposing: $f_{1}=\frac{Q_{i n}}{B}-f_{0}-f_{2}$. The outflow water height is carried out by $f_{2}=h_{0}-f_{0}-f_{1}$. But this solution will create a rapid change in $f_{2}$ and cause oscillations for the discharge at the right extremity. So we use the following relaxation algorithm:

- Calculate the new value of $f_{2}$ as: $f_{2}=h_{0}-f_{0}-f_{1}$.

- Calculate the new discharge: $q=v\left(f_{1}-f_{2}\right)$

- Calculate the true discharge at the outflow-end: $q^{t}=(1-\alpha) q+\alpha q^{t-\Delta t}$ where $\alpha \in[0 ; 1]$

- Re-calculate the value of $f_{2}: f_{2}=f_{1}-\frac{q^{t}}{v}$

\subsection{Simulation}

With all three methods (LB, Preissmann, FV) a simulation is run until a steady flow is reached. Different numbers of points, $N$, are used to test the precision. For each $N$, the spatial step is defined as $\Delta x=\frac{L}{N}$ and the time step, $\Delta t=\frac{\Delta x}{v}$, where $v$ is fixed $(v=2)$. These values are used for all three methods. We consider that the steady solution $h_{s}$ is attained if $e<10^{-8}$ where $e$ is the relative distance of the water height profile between two consecutive iterations. It is calculated as:

$$
e=\frac{\left\|h_{t}-h_{t-\Delta t}\right\|}{\left\|h_{t}\right\|}
$$

with $\|x\|=\sqrt{\sum_{i} x_{i}^{2}}$. The relative error, $\epsilon$, is calculated as:

$$
\epsilon=\frac{\left\|h_{s}-h_{r e f}\right\|}{\left\|h_{r e f}\right\|}
$$




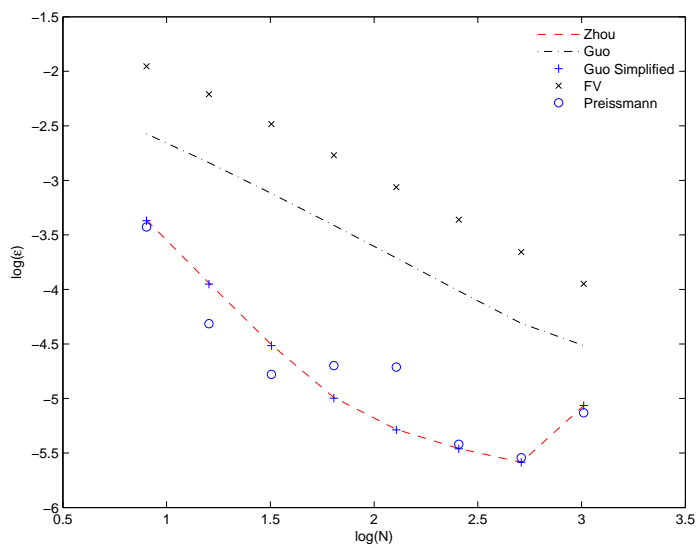

Figure 12: Precision of different methods.

The CPU time, $T_{C P U}$, is determined as the time to reach the steady solution. The results are displayed in (Fig.12) and (Fig.13). The Zhou's force model and Guo's simplified force term (the latter was obtained in section 3.2 with $A=0$ and $B=\left(1-\frac{1}{2 \tau}\right) F$ ) behave identically. Both exhibit second-order accuracy from 8 to 512 grid points before showing an error increase when $N=1024$. This suggests that the convective scaling $\Delta t \propto \Delta x$ we used should be replaced by a diffusing scaling $\Delta t \propto \Delta x^{2}$ when $\Delta x$ becomes small.

The Guo force term is only first order accurate and is also less precise in magnitude. The Preissmann scheme has a same performance as Zhou's force term, except for $N=64$ and $N=128$. In these cases, the steady solution is reached with $e=0$, so $\epsilon$ can not be reduced.

Finally, the finite volume method is first order (as expected), and is the least accurate.

We previously mentioned that Guo's force term leads to an implicit definition of $u$ while Zhou's force term does not. In calculating the CPU time, we used Zhou's force term.

For all methods, the CPU time increases by a factor of 4 when the number of points is doubled, because the number of iterations is also doubled to reach the steady solution (due to our scaling of $\Delta t$ versus $\Delta x$ ). We observe that the LB solver is much faster than the two others. It is more than 100 times faster than the Preismann methods and about 10 times faster than the FV approach. This fact can be explained by examinating in detail the calculation of each scheme. For the LB method, at each time step, just $f^{e q}$ and $f^{\text {out }}$ need to be evaluated to obtain $h$ and $u$. For the FV approach, we have to solve a series of local Riemann problems and the source term is calculated by point-implicitly method (see [19]), which results great amount of operators to execute. In Preissmann schema, a iteration method is use to find the solution of a system of $N$ non-linear equation which requires a great number of evaluation of these equations. As a result, this 


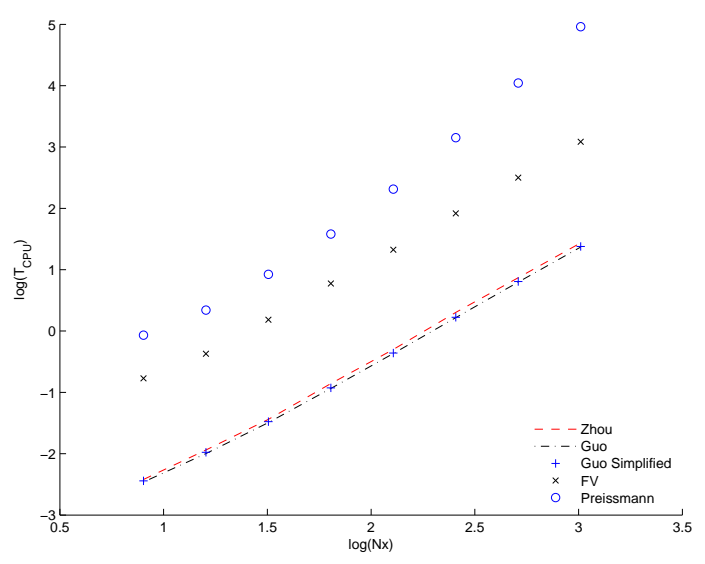

Figure 13: CPU calculation time

schema is far slower than the two others.

Finally we consider the numerical stability of these three schemes. The Preissmann scheme is implicit and unconditionally stable. The stability region of the LBM is limited by $\mathrm{Fr}<1$ and $\mathrm{Fr}<\frac{1}{\phi}-1$ as indicated in (Fig.7). This conclusion can be verified by the simulation, as follows.

We consider a long canal of length, $L=8 m$, with no slope and no friction. We impose periodic boundary conditions by setting $f_{1}^{\text {in }}\left(x_{1}\right)=f_{1}^{\text {out }}\left(x_{N}\right)$ and $f_{2}^{\text {in }}\left(x_{N}\right)=f_{2}^{\text {out }}\left(x_{1}\right)$ (see Fig.11). We initialize this canal with a water height perturbed as $h(x, 0)=h_{0}+0.1 e^{-\frac{(x-4)^{2}}{0.1}}$ with $h_{0}=0.1 \mathrm{~m}$. An initial speed $u$ is imposed according to the chosen Froude number, according to the relation $u=\operatorname{Fr} \sqrt{g h_{0}}$. In order to choose $\phi$ the lattice speed $v$ is adjusted as $v=\frac{\sqrt{g h_{0}}}{\phi}$. We do this simulation with different values of $\tau$.

The stability of the system is tested by using the entropies notion [17]. Entropies of the system (1-2) without friction and slope are functions $(h, u) \rightarrow$ $E(h, u)$ such that for some function $F:(h, u) \rightarrow(h, u)$, called the entropy flux, we have:

$$
\frac{\partial F}{\partial h}=u \frac{\partial E}{\partial h}+g \frac{\partial E}{\partial u} \text { and } \frac{\partial F}{\partial u}=h \frac{\partial E}{\partial h}+u \frac{\partial E}{\partial u} .
$$

With these functions, if we let $R=\int_{0}^{L} E(h, u) d x$ then we have $\dot{R}=-[F(h, u)]_{0}^{L}=$ 0 because of the periodic boundary condition. This means that $R$ is a conserved scalar quantity which can be used to test the stability of the integration scheme. A possible entropy function for the shallow water equations $(1,2)$ is introduced in $[7]$ :

$$
E(h, u)=\frac{1}{2} h u^{2}+\frac{1}{2} g h^{2}, \quad F(h, u)=\frac{1}{2} h u^{3}+g h^{2} u
$$

We will consider that the considered discretization scheme is stable if after 10000 iterations the entropy value has not exceeded $1 \%$ of its initial value. We follow 
the same procedure for the FV method, but this time, taking into account only Fr and $\phi$, since $\tau$ is not a parameter of the FV scheme. The results are presented in Fig.14.

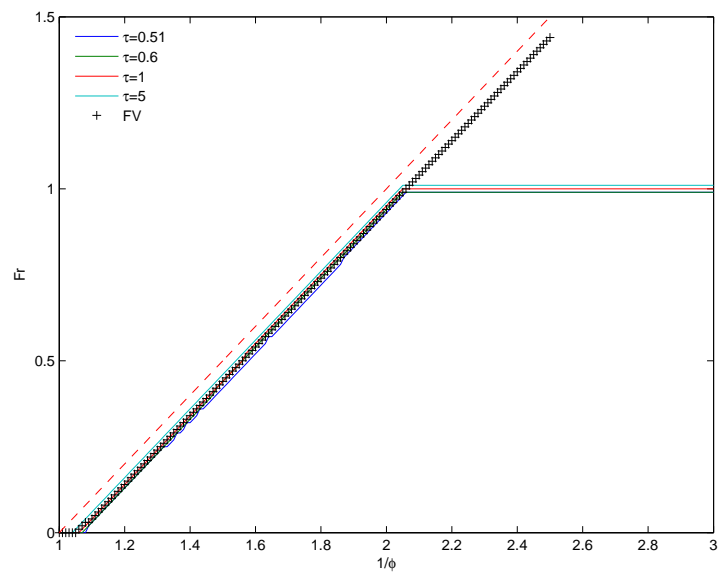

Figure 14: The stability region of LBM (lines) and finite volume method $(+)$. The Courant condition is presented as a dashed line. There is a slight difference with Fig. 7 because the simulation is non-linear.

As expected, the limits of stability of the LB method are the Courant condition and Fr $<1$. However, we can modify the present D1Q3 model to obtain a new model which is stable for both Fr $<1$ and $\mathrm{Fr}>1$ as is described in [3].

\section{Coupling experiments}

In this section, we show how to use the 1D shallow water LB model to simulate a complex canal structure by coupling two or more canal sections. We will first consider many examples of hydraulic works like submerged gates, pumping stations, spillways, branching junctions or mixed interconnection structures. Then we will apply the proposed coupling methodology (and the 1D shallow water LB model for single reaches) to develop the full model of a real example: the Canal de la Bourne network which irrigates the East of Valence (Drôme, France) agricultural plains with the water from the Vercors Mountains. Finally some numerical simulations will prove the efficiency of the proposed methodology.

\subsection{Coupling relations}

When coupling two 1D canal sections that are described with a LB model, some of the $f_{i}$ 's are known and other are unknown at the junction. In order to connect two segments coupled by a gate or a pump station, one has to compute, for each segment, the missing distributions. Referring to Fig. 15 we denote 


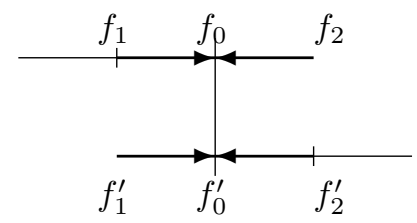

Figure 15: The known $\left(f_{2}^{\prime}, f_{0}^{\prime}\right.$ and $\left.f_{1}, f_{0}\right)$ and unknown $\left(f_{2}\right.$ and $\left.f_{1}^{\prime}\right)$ distributions $f_{i}$ at a connection point.

by $f_{i}$ the density distributions of the up-stream system, and by $f_{i}^{\prime}$ the density distributions of the down-stream system. The unknown variables are then $f_{2}$ and $f_{1}^{\prime}$. They can be obtained by solving an equation describing the physical properties of the coupling.

\subsubsection{Connection through a gate}

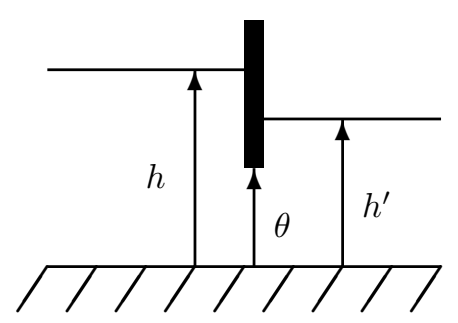

Figure 16: Gate structure

A gate in a submerged regime is presented in Fig. 16. The flow rate $Q$ through the gate is governed by the difference between the up-stream water level $h$ and down-stream level $h^{\prime}$, and is given by the well known gate eq. [8]

$$
Q=B_{g} \alpha \theta \sqrt{2 g\left(h-h^{\prime}\right)}
$$

where $B_{g}$ is the gate width, $\alpha$ the gate coefficient, $\theta$ the gate opening and $g$ the gravity.

The coupling through a gate imposes that the flows $Q$ and $Q^{\prime}$ are the same. From the definition of the water level and the discharge, we have the relations:

$$
\begin{aligned}
& h=f_{0}+f_{1}+f_{2} \\
& h^{\prime}=f_{0}^{\prime}+f_{1}^{\prime}+f_{2}^{\prime} \\
& Q=v B\left(f_{1}-f_{2}\right)=v B^{\prime}\left(f_{1}^{\prime}-f_{2}^{\prime}\right)=B_{g} \alpha \theta \sqrt{2 g\left(h-h^{\prime}\right)}
\end{aligned}
$$

where $B$ denotes the width at up-stream and $B^{\prime}$ the width at down-stream of the gate. So we obtain a system of two equations that we can solve for $f_{2}$ and 
$f_{1}^{\prime}$

$$
\left\{\begin{array}{l}
B\left(f_{1}-f_{2}\right)=B^{\prime}\left(f_{1}^{\prime}-f_{2}^{\prime}\right) \\
v B\left(f_{1}-f_{2}\right)=B_{g} \alpha \theta \sqrt{2 g} \sqrt{\left(f_{0}+f_{1}+f_{2}\right)-\left(f_{0}^{\prime}+f_{1}^{\prime}+f_{2}^{\prime}\right)}
\end{array}\right.
$$

From the first equation, we have $f_{1}^{\prime}=B / B^{\prime}\left(f_{1}-f_{2}\right)+f_{2}^{\prime}$. By replacing this in the second equation, we have:

$$
f_{1}-f_{2}=k \sqrt{r+f_{2}\left(1+\frac{B}{B^{\prime}}\right)}, k=\frac{B_{g} \alpha \theta \sqrt{2 g}}{v B}, r=f_{0}-f_{0}^{\prime}+f_{1}\left(1-\frac{B}{B^{\prime}}\right)-2 f_{2}^{\prime}
$$

which is equivalent to

$$
f_{2}^{2}-\left(2 f_{1}+\left(1+B / B^{\prime}\right) k^{2}\right) f_{2}+f_{1}^{2}-k^{2} r=0
$$

This is a second-order equation in $f_{2}$ and we take the positive solution:

$$
f_{2}=\frac{1}{2}\left(2 f_{1}+\left(1+B / B^{\prime}\right) k^{2}+k \sqrt{4\left(1+B / B^{\prime}\right) f_{1}+\left(1+B / B^{\prime}\right)^{2} k^{2}+4 r}\right)
$$

And $f_{1}^{\prime}$ is calculated by:

$$
f_{1}^{\prime}=B / B^{\prime}\left(f_{1}-f_{2}\right)+f_{2}^{\prime}
$$

\subsubsection{Connection by a pumping station}

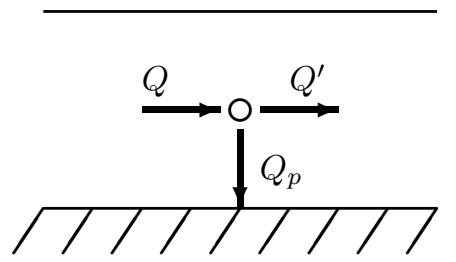

Figure 17: Schematic description of a pumping station

A pumping station is illustrated in Fig. 17. The relation between the flow rate, $Q$, and water level, $h$, before the pump and $Q^{\prime}$ and $h^{\prime}$ after the pump are that

$$
\begin{aligned}
& Q=Q^{\prime}+Q_{p} \\
& h=h^{\prime}
\end{aligned}
$$

where $Q_{p}$ is the flow rate taken by the pump.

Using the same notation as introduced in Fig. 15 and from the definition of the water depth and the flow rate we obtain

$$
\Rightarrow\left\{\begin{array}{l}
v B\left(f_{1}-f_{2}\right)=v B^{\prime}\left(f_{1}^{\prime}-f_{2}^{\prime}\right)+Q_{p} \\
f_{0}+f_{1}+f_{2}=f_{0}^{\prime}+f_{1}^{\prime}+f_{2}^{\prime} \\
f_{2}=\frac{1}{1+B / B^{\prime}}\left(2 f_{2}^{\prime}+f_{0}^{\prime}-f_{0}+\left(B / B^{\prime}-1\right) f_{1}-\frac{Q_{p}}{v B}\right) \\
f_{1}^{\prime}=\frac{1}{2+B^{\prime} / B}\left(2 f_{1}+f_{0}-f_{0}^{\prime}+\left(B^{\prime} / B-1\right) f_{2}^{\prime}-\frac{Q_{p}}{v B}\right)
\end{array}\right.
$$




\subsubsection{Connection through a spillway}

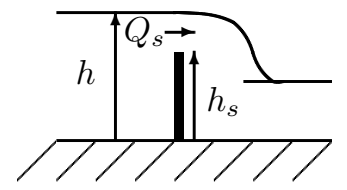

Figure 18: Spillway structure

Spillways, such as represented in Fig. 18, are commonly used in irrigation networks to guarantee (stabilize) the water level in the upstream part whatever the water flow is. The flow rate through the spillway is given by [8]:

$$
Q_{s}=\left\{\begin{array}{c}
L_{s} R_{s} \sqrt{2 g\left(h-h_{s}\right)^{3}}, \text { if } h \geq h_{s} \\
0 \text { if } h<h_{s}
\end{array}\right.
$$

where $L_{s}$ is the spillway's width, $R_{s}$, the spillway's coefficient, $h$, the water level at up-stream and, $h_{s}$, the spillway's height. By the definition of $h$ and $Q$, we have:

$$
v B\left(f_{1}-f_{2}\right)=\left\{\begin{array}{c}
L_{s} R_{s} \sqrt{2 g\left(f_{0}+f_{1}+f_{2}-h_{s}\right)^{3}}, \text { if } f_{0}+f_{1}+f_{2} \geq h_{s} \\
0 \text { if } f_{0}+f_{1}+f_{2}<h_{s} .
\end{array}\right.
$$

This nonlinear equation can be solved by any numerical method to obtain the unknown $f_{2}$. More complex models for the spillway can be used without modifying the general structure of these constitutive equations. Again the other unknown $f_{1}^{\prime}$ is determined using the water conservation assumption by eq. (118).

\subsubsection{Branching canal}

We can also define the coupling relation in the case of a canal that splits in two branches. In terms of the distributions $f_{i}$, the situation is illustrated in Fig. 19. An up-stream section with distributions $f$ meets two down-stream sections described with distribution functions $f^{\prime}$ and $f^{\prime \prime}$, respectively.

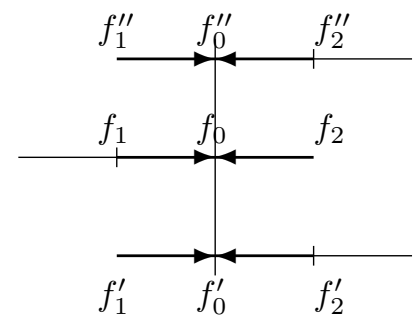

Figure 19: The known $\left(f_{2}^{\prime}, f_{0}^{\prime}, f_{2}^{\prime \prime}, f_{0}^{\prime \prime}\right.$ and $\left.f_{1}, f_{0}\right)$ and unknown $\left(f_{2}, f_{1}^{\prime}\right.$ and $\left.f_{1}^{\prime \prime}\right)$ distributions $f_{i}$ at a connection point.

At the branching, the water height is the same for the three branches, whereas the up-stream discharge is divided in two parts. Thus, the following 
relations have to be satisfied

$$
\begin{gathered}
h=h^{\prime}=h^{\prime \prime} \\
Q=Q^{\prime}+Q^{\prime \prime}
\end{gathered}
$$

Where $h, Q$ are water height and discharge in the up-stream canal at the junction, while $h^{\prime}, Q^{\prime}$, and $h^{\prime \prime}, Q^{\prime \prime}$ are water heights and discharges in the two downstream branches.

The above three equations can be expressed in terms of the distributions

$$
\left\{\begin{array}{l}
f_{0}+f_{1}+f_{2}=f_{0}^{\prime}+f_{1}^{\prime}+f_{2}^{\prime} \\
f_{0}+f_{1}+f_{2}=f_{0}^{\prime \prime}+f_{1}^{\prime \prime}+f_{2}^{\prime \prime} \\
v B\left(f_{1}-f_{2}\right)=v B^{\prime}\left(f_{1}^{\prime}-f_{2}^{\prime}\right)+v B^{\prime \prime}\left(f_{1}^{\prime \prime}-f_{2}^{\prime \prime}\right)
\end{array}\right.
$$

where $B, B^{\prime}$, and $B^{\prime \prime}$ denote the canal's width in the three branches. After isolating the unknown distributions, this systems becomes

$$
\left\{\begin{aligned}
f_{2}-f_{1}^{\prime} & =-f_{0}-f_{1}+f_{0}^{\prime}+f_{2}^{\prime} \\
f_{2}-f_{1}^{\prime \prime} & =-f_{0}-f_{1}+f_{0}^{\prime \prime}+f_{2}^{\prime \prime} \\
B f_{2}+B^{\prime} f_{1}^{\prime}+B^{\prime \prime} f_{1}^{\prime \prime} & =B f_{1}+B^{\prime} f_{2}^{\prime}+B^{\prime \prime} f_{2}^{\prime \prime}
\end{aligned}\right.
$$

which can be solved as

$$
\begin{aligned}
& f_{2}=\frac{1}{K}\left[-\left(B^{\prime}+B^{\prime \prime}\right) f_{0}+\left(B-B^{\prime}-B^{\prime \prime}\right) f_{1}+B^{\prime} f_{0}^{\prime}+2 B^{\prime} f_{2}^{\prime}+B^{\prime \prime} f_{0}^{\prime \prime}+2 B^{\prime \prime} f_{2}^{\prime \prime}\right] \\
& f_{1}^{\prime}=\frac{1}{K}\left[B f_{0}+2 B f_{1}-\left(B+B^{\prime \prime}\right) f_{0}^{\prime}+\left(B-B^{\prime}+B^{\prime \prime}\right) f_{2}^{\prime}+B^{\prime \prime} f_{0}^{\prime \prime}+2 B^{\prime \prime} f_{2}^{\prime \prime}\right] \\
& f_{1}^{\prime \prime}=\frac{1}{K}\left[B f_{0}+2 B f_{1}+B^{\prime} f_{0}^{\prime}+2 B^{\prime} f_{2}^{\prime}-\left(B+B^{\prime}\right) f_{0}^{\prime \prime}+\left(-B-B^{\prime}+B^{\prime \prime}\right) f_{2}^{\prime \prime}\right]
\end{aligned}
$$

where $K=B+B^{\prime}+B^{\prime \prime}$

\subsubsection{Connection through a mixed structure}

Finally, we show how to connect two canal sections with a mixed structure consisting of different elements placed in parallel (such as two gates side by side, a gate and a spillway, etc.). We present here the case of two gates and one spillway as represented in Fig. 20. The other cases can be treated similarly.

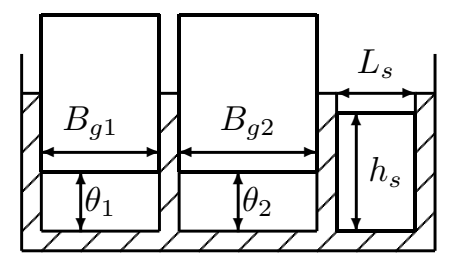

Figure 20: Structure with 2 gates and 1 spillway in parallel.

The flow rate is determined by:

$$
\begin{aligned}
Q & =Q_{g 1}+Q_{g 2}+Q_{s} \\
Q_{g_{1}} & =\alpha_{1} B_{g 1} \theta_{1} \sqrt{2 g\left(h-h^{\prime}\right)} \\
Q_{g_{2}} & =\alpha_{2} B_{g 2} \theta_{2} \sqrt{2 g\left(h-h^{\prime}\right)} \\
Q_{s} & =\left\{\begin{array}{c}
L_{s} R_{s} \sqrt{2 g\left(h-h_{s}\right)^{3}}, \text { if } h \geq h_{s} \\
0 \text { if } h<h_{s}
\end{array}\right.
\end{aligned}
$$


As in previous sections, we have the following relations:

$$
\begin{aligned}
Q & =v B\left(f_{1}-f_{2}\right)=v B^{\prime}\left(f_{1}^{\prime}-f_{2}^{\prime}\right) \\
h & =f_{0}+f_{1}+f_{2} \\
h^{\prime} & =f_{0}^{\prime}+f_{1}^{\prime}+f_{2}^{\prime}
\end{aligned}
$$

Again, these equations need to be solved by a numerical method to obtain the unknowns $f_{2}$ and $f_{1}^{\prime}$.

\subsection{Simulation example}

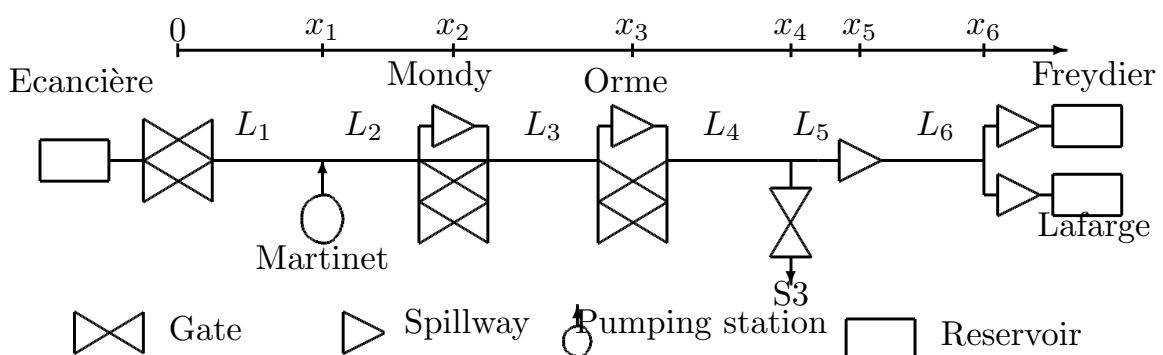

Figure 21: Structure of the Bourne irrigation system

Our simulation example is a model of the canal de la Bourne irrigation network. This network was built in the late 19th century to irrigate the plains around Valence in France. It is still in use now and its fine modelling and control has become a new challenge. Indeed, the demand on water considerably increased these last few decades as, more recently, the constraints on the quantity of water which may be withdrawn from the upstream natural river La Bourne became more and more binding limitations. A sketch of the canal network is presented in Fig. 21. The main reach (from $x=0$ to $x=x_{6}$ ) is about $30 \mathrm{~km}$ long. The main irrigation network consists of:

- An upstream reservoir at Ecancière which supplies the canal through two gates and has a constant water level.

- A pumping station at Martinet which pumps water from Isère river to the canal or can produce electricity during the Autumn and Winter seasons.

- A gate at Mondy which consists of two submerged gates and a spillway (see the mixed interconnection structure developed in the previous section).

- A gate at Orme with the same structure as the one in Mondy.

- A Secondary canal, termed S3, which takes water from the canal through a submerged gate.

- A spillway, just after S3, which aims at maintaining the water supply for S3. 
- Two reservoirs at Lafarge and Freydier which receive the water from the canal through spillways.

To simulate this system, we divide it into 6 segments and use the previously presented methods to connect them. The parameters used are presented in Tables 1-3. The lattice Boltzmann model is carried out with $\Delta x=100(\mathrm{~m})$, $\Delta t=40(s)$ and $\tau=0.7$;

\begin{tabular}{|c|c|c|c|c|c|c|}
\hline & $L_{1}$ & $L_{2}$ & $L_{3}$ & $L_{4}$ & $L_{5}$ & $L_{6}$ \\
\hline Length $(\mathrm{m})$ & 5900 & 6900 & 2650 & 7000 & 100 & 3325 \\
\hline Slope $\left(10^{-4}\right)$ & 2,753 & 2,753 & 2,441 & 2,4 & 2,4 & 2,4 \\
\hline Width $(\mathrm{m})$ & 5,605 & 5,605 & 5,1 & 4,36 & 4,36 & 4,36 \\
\hline Manning coefficient & \multicolumn{7}{|c|}{0.033} \\
\hline
\end{tabular}

Table 1: Parameters for the reaches

\begin{tabular}{|c|c|c|c|c|}
\hline & $\alpha_{1}$ & $\alpha_{2}$ & $B_{1}(\mathrm{~m})$ & $B_{2}(\mathrm{~m})$ \\
\hline Ecancière & & & 2,4 & 2,4 \\
Mondy & 0,66 & 0,66 & 1,07 & 2,9 \\
Orme & & & 2,9 & 2,9 \\
\hline Gate in S3 & 0,66 & & 2 & \\
\hline
\end{tabular}

Table 2: Parameters for the gates

\begin{tabular}{|c|c|c|c|}
\hline & $R_{s}$ & $L_{s}(\mathrm{~m})$ & $h_{s}(\mathrm{~m})$ \\
\hline Mondy & & 0,8 & 1,3 \\
Orme & & 1,91 & 1,7 \\
Spillway S3 & 0.35 & 9,6 & 0,8 \\
Lafarge & & 4 & 1,35 \\
Freydier & & 2 & 1,57 \\
\hline
\end{tabular}

Table 3: Parameters of the spillways

The model has first to be initialized. From the shallow water equations $(1,2)$, it could be noticed that the water flow equilibrium profile is necessarily uniform while the water level profile is generally non-uniform (unless the condition $I=J$ holds which is the case only when the friction forces precisely equilibrate the "gravity" forces). The steady state initial profiles are thus constructed in the following way:

- Choose the water height $h\left(x_{6}^{+}\right)$and calculate the flow rate of the steady state $Q_{0}=Q\left(x_{6}\right)$

- Integrate eq. (103) to obtain the water height $h(x), x \in\left[x_{5}, x_{6}\right]$ with the boundary condition at $x_{6}$ 
- Calculate the water height up-stream of the spillway S3 $h\left(x_{5}^{+}\right)$by imposing the discharge through the spillway $Q_{0}$

- Integrate eq. (103) to obtain the water height $h(x), x \in\left[x_{4}, x_{5}\right]$ with the boundary condition at $x_{5}^{+}$

- Choose the flow rate withdrawn by the secondary canal S3 $Q_{S 3}$ and integrate eq. (103) to obtain the water height $h(x), x \in\left[x_{3}, x_{4}\right]$ with the boundary condition at $x_{4}^{+}: h\left(x_{4}^{+}\right)=h\left(x_{4}^{-}\right)$and the discharge $Q_{1}=$ $Q_{0}+Q_{S 3}$

- Choose the water height at $x_{3}^{+}$and determine the gates opening by using the discharge $Q_{1}$, the gate equations and the spillway equations.

- Integrate eq. (103) to obtain the water height $h(x), x \in\left[x_{2}, x_{3}\right]$ with the boundary condition at $x_{3}^{+}$

- Choose the water height at $x_{2}^{+}$and determine the gates opening by using the discharge $Q_{1}$, the gate equations and the spillway equations.

- Integrate eq. (103) to obtain the water height $h(x), x \in\left[x_{1}, x_{2}\right]$ with the boundary condition at $x_{2}^{+}$

- Choose the discharge supplied by the pump station $Q_{p}$

- Integrate eq. (103) to obtain the water height $h(x), x \in\left[0, x_{1}\right]$ with the boundary condition at $x_{2}^{+}: h\left(x_{2}^{+}\right)=h\left(x_{2}^{-}\right)$and the discharge $Q_{2}=Q_{1}-Q_{p}$

- Use the boudary condition at $x_{4}$ and the discharge $Q_{S 3}$ to initialize the secondary canal S3.

These initial conditions are presented in Fig. 22 and Fig. 23. Starting with
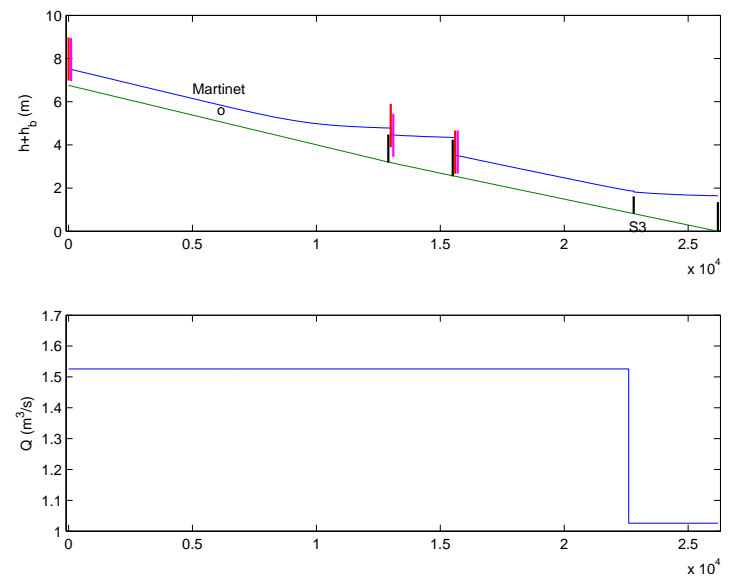

Figure 22: Initial conditions for the principal canal 

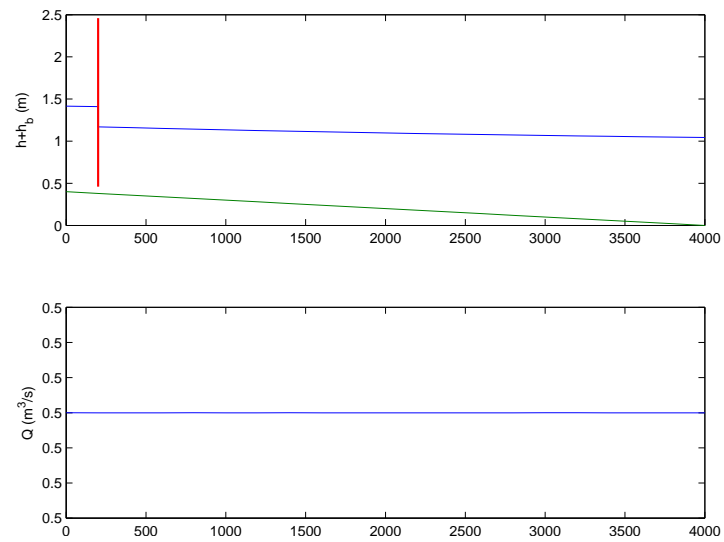

Figure 23: Initial conditions for the secondary canal

this equilibrium configuration for the irrigation network, we use the following scenario for the simulation of the transient behaviour of the model. Firstly, at time $t=0(h)$ the opening of the first gate at Ecancière increases by $90 \%$, following a ramp, to reach its final value with a rate of $5.6 \times 10^{-4}(\% / s)$. Then at time $t=1(h)$ the pumping station at Martinet starts to withdraw water from the canal. The withdrawal flow rate increases with a rate of $0.02\left(\mathrm{~m}^{3} / \mathrm{s}\right)$ to reach its final value at $0.5\left(\mathrm{~m}^{3} / \mathrm{s}\right)$. Finally, at time $t=2(h)$, the gate opening in S3 increases by $30 \%$, following a ramp, to reach its final value with the same speed as for the gate at Ecancière. The simulation results are presented in Fig. 24 and Fig. 25. We can see the wave propagation phenomenon with constant speed and the discharge discontinuity corresponding to withdrawal at the pumping station and in the secondary canal. These simulation results agree with the measured values for this scenario.

Finally, from a complexity point of view, it could be noticed that with the computer used to perform this simulation (with a code written in Matlab) it is only possible to update up to $1.3 \times 10^{4}$ sites per second. A simulation over 1 year for the above network described with 159 sites requires about 2.5 hours of CPU times. A C++ implementation is typically 100 times faster, without any code optimization, and reduces the time needed for this 1-year simulation (with the same spatial and temporal resolutions) to the order of a few minutes of CPU times. For real scale irrigation networks we are thus far beyond real time requirements. However this efficiency may be useful for reduced scale experimental micro-canals (with typically fast dynamics associated with low frictions and short reaches which are only a few meters long, rather than a few kilometers long), for complex irrigation networks (with a complex tree of secondary canals connected trough dozens of hydraulic works, many pumping stations or reservoirs, etc.) or for other fluid flow application examples for the ideas presented in this paper. 

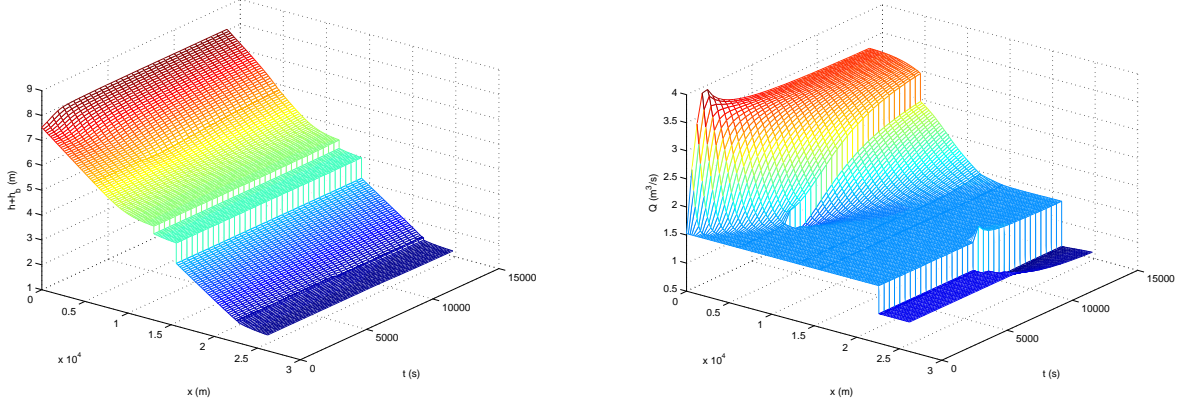

Figure 24: Evolution in the main canal
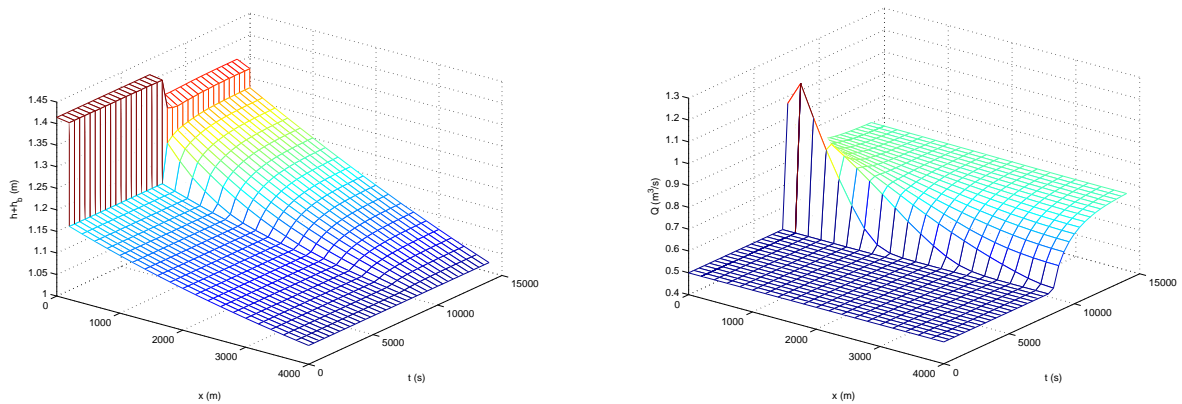

Figure 25: Evolution in the secondary canal 


\section{Conclusions}

In this work, we discuss the capability of the Lattice Boltzmann method to solve the 1D shallow water equation. We proposed an exact analytical study of its accuracy and its stability when the system is linearized around a water level, $h$, and a velocity, $u$. We derived the dissipative term as well as the nonequilibrium part of the distribution function, $f_{i}$. Our derivation is validated by numerical simulations. Our results show that the viscosity term reported in some recent publications on the LB method for the shallow water equations has been incorrectly calculated. We also propose a detailed analysis of the way to add an external force on the LB model. Exact analytical solutions of the LB model with force have been obtained in a simple situation.

Furthermore we compare the 1D LB model with two other solvers: an implicit finite difference scheme and a finite volume approach. Our comparison showed that the LB model is more precise and significantly faster than the other methods. However the stability region of the LB model is limited by the Courant condition and sub-critical flow conditions, unless some model extensions are considered, such as the asymmetric D1Q3 LB model which can correctly describe the transition between the fluvial and torrential regimes.

A coupling methodology to interconnect several 1D models is also developed. It is found to give very good results. Therefore our coupling strategy allows us to simulate many canal sections interconnected through different types of structures (e.g. gates, spillways, pumping stations or branchings).

Other coupling problems, such as the coupling between a $1 \mathrm{D}$ and a $2 \mathrm{D}$ LB shallow water models, or between two 1D models with different resolutions are also possible although not described here. See [21] for more details. The coupling of LB shallow water models with a fully resolved, free surface flow model, such as that developed in $[12,13]$, is currently under investigation and will be reported in a forthcoming publication.

All these results show the promising potential of the LB approach to simulate a realistic complex network of irrigation canals.

\section{Acknowledgments}

We thank Guy Simpson for providing us with the finite volume solver used in this study.

\section{References}

[1] S. Chen and G.D. Doolen. Lattice Boltzmann methods for fluid flows. Annu. Rev. Fluid Mech., 30:329, 1998.

[2] B. Chopard and M. Droz. Cellular automata modeling of physical systems. Cambridge University Press, 1998. 
[3] Bastien Chopard, Pham van Thang, and Laurent Lefèvre. An asymmetric lattice boltzmann model for the $1 \mathrm{~d}$ shallow water equation. Communication in Computational Physics, 2009. submitted.

[4] V.T. Chow. Open channel hydraulics. Mc Graw Hill, New-York, 1985.

[5] P.J. Dellar. Nonhydrodynamic modes and a priori construction of shallow water lattice boltzmann equation. Phys. Rev. E, 65:036309, 2002.

[6] J.B. Frandsen. A simple lbe run-up model. Progress in Computational Fluid Dynamics, 8:222-232, 2008.

[7] D. Georges and X. Litrico (Eds.). Automatique pour la gestion des ressources en eau. Hermès, 2002.

[8] W.H. Graf and M.S. Altinakar. Hydraulique fluviale - Ecoulement et phénomènes de transport dans les canaux â géométrie simple, volume 16 of Traité de génie civil de l'Ecole Polytechnique Fédérale de Lausanne. Presses Polytechniques Universitaires Romandes, 2000. ISBN 978-2-88074-812-8.

[9] Zhaoli Guo, Chguang Zheng, and Baochang Shi. Discrete lattice effects on forcing terms in the lattice Boltzmann method. Phys. Rev. E, 65:046308, 2002 .

[10] Xiaoyi He, Xiaowen Shan, and Gary D. Doolen. Discrete Boltzmann equation model for nonindeal gases. Phys. Rev. E, 57:R13-R16, 1998.

[11] A.L. Kupershtokh. A new method of incorporating a body force term into a lattice Boltzmann equation. In France University of Poitier, editor, 5ème Congrès International d'Electrodynamique, pages 241-246, 2004.

[12] O. Marcou, B. Chopard, and S. El Yacoubi. Modeling of irrigation canals: a comparative study. Int. J. Mod. Phys. C, 18(4):739-748, 2007.

[13] O. Marcou, B. Chopard, S. El Yacoubi, B. Hamroun, L. Lefèvre, and E. Mendes. Lattice Boltzmann models for simulation and control of unsteady flows in open channels. J. of Irr. and Drain. Eng., 2010. in press.

[14] O. Marcou, S. El Yacoubi, and B. Chopard. A bi-fluid Lattice Boltzmann model for water flow in an irrigation channel. In International Conference on Cellular Automata for Research and Industry, number 7, pages 373-382, Perpignan, France, September 2006.

[15] Y.H. Qian, D. d'Humières, and P. Lallemand. Lattice BGK models for Navier-Stokes equation. Europhys. Lett., 17(6):479-484, 1992.

[16] R. Salmon. The lattice Boltzmann method as a basis for ocean circulation modeling. J. Marine Research, 57:503-535, 1999.

[17] Denis Serre. Systèmes de lois de conservation I. Diderot Editeur, Arts et Sciences, 1996. 
[18] Xiaowen Shan and Hudong Chen. Lattice Boltzmann model for simulating flows with multiple phases and components. Phys. Rev. E, 47:1815-1819, 1993.

[19] Guy Simpson and Sébastien Castelltort. Coupled model of surface water flow, sediment transport and morphological evolution. Computers $\mathscr{E}$ Geosciences, 32:1600-1614, 2006.

[20] Sauro Succi. The Lattice Boltzmann Equation, For Fluid Dynamics and Beyond. Oxford University Press, 2001.

[21] Pham van Thang. Modélisation et commande des systèmes non-linéaire à paramètres distribués par la méthode de Boltzmann sur réseau: application aux canaux d'irrigation. Technical report, Grenoble INP ESISAR, France, 2009. Master's dissertation.

[22] J.G. Zhou. Lattice Boltzmann methods for shallow water flows. Springer, 2004. 